\title{
THEORETISCHE ÜBERLEGUNGEN UND HISTORISCHE SCHLAGLICHTER ZUM THEMA »EROTIK UND MUSIK«
}

\section{Dietrich Helms}

Am Anfang war die Musik - und dann der Sex. So muss es zumindest gewesen sein, glaubt man manchen Evolutionsbiologen und Anthropologen. Bereits Charles Darwin behauptete in Der Ausdruck der Gemütsbewegung bei dem Menschen und den Tieren (1910: 76ff.), der Ursprung der Musik liege in den Balzlauten der Tiere, deren Gesang Mittel der natürlichen Zuchtwahl sei. In jüngster Zeit ist diese These - übertragen auf Hominiden und mit Ergebnissen archäologischer und anthropologischer Studien begründet - wieder sehr beliebt (vgl. Gembris 2005; Wallin et al. 2000). So diskutiert Steven Mithen, zugespitzt formuliert, die These, dass The Singing Neanderthals (2006) die Musik entwickelt hätten, um Weibchen anzulocken. Wenn dem so wäre, erklärte das natürlich einiges: warum in fast allen Kulturen gesungen wird, warum das Musikbusiness von Männern beherrscht wird und warum hässliche, alte Rock-Musiker anscheinend immer die schönsten, jungen Frauen abbekommen.

Allerdings stimmt bereits der Versuch einiger Autoren kritisch, die männliche Dominanz des Musikgeschäfts als Beleg für die Zuchtwahlthese anzuführen (vgl. ebd.: 180). Sind es nicht in Mythologie und Geschichten immer sirenenhafte Frauen, die Männer durch ihren Gesang vom rechten Kurs und Weg abbringen? Und es erstaunt doch sehr, dass musikalische Fähigkeiten, wenn sie denn tatsächlich ein Selektionsmerkmal wären, ausgerechnet bei den Alphamännchen und -weibchen unserer Gesellschaft, bei Topmanagern und Spitzenpolitikern, nicht überdurchschnittlich verbreitet sind, und die Fortpflanzungsrate ausgerechnet bei den Heroen unserer Musikgeschichten - sieht man einmal von Johann Sebastian Bach und Richard Wagner ab - eher unterdurchschnittlich ist. Man stelle sich vor, welche Bedeutung die Musikpädagogik hätte, wenn nur die besten Musiker zur Fortpflanzung kämen.

Wirklich problematisch ist die Behauptung, der Ursprung der Musik sei im Balzverhalten zu suchen, vor allem durch die Verwendung des Begriffes 
»Musik«. Die These Darwins erscheint doch nur deshalb beim ersten Lesen sinnvoll, weil wir uns daran gewöhnt haben, dass die Lautäußerungen von Vögeln mit der Metapher des Gesangs bezeichnet werden und neuerdings auch die Lautäußerungen von Walen gleich neben dem Wähnen Wagnerscher Walküren im CD-Regal zu finden sind. Bezeichnet man Vogelgesänge und Walgezwitscher pauschal als »Musik«, sind alle akustischen Lebensäußerungen aus der Fauna »Musik«: »El grillo é boun cantore« komponierte Josquin Desprez - allerdings ohne es allzu ernst zu meinen. Damit wären aber auch das Bellen des Hundes, die Sprache des Menschen »Musik« und unter den Verallgemeinerungen wäre all das verschüttet, was Musik gerade zur Musik macht.

Ich meine, Musik ist eine kulturelle Kategorie. Die natürliche Zuchtwahl Darwins dagegen ist eine biologische Kategorie. Beides lässt sich kaum glaubwürdig zueinander in Beziehung setzen, will man nicht alle Äußerungen des Lebens auf den Überlebenskampf der Gene reduzieren und die Biologie als Wissenschaft vom Leben zur Wissenschaft aller Lebensäußerungen erheben. Was nicht zu leugnen ist: Teile unseres immer weiter ausufernden Musikbegriffs lassen sich als Elemente kommunikativer Systeme beschreiben: »Musik « meint (auch) Kommunikation. Als solche lässt sie sich in Beziehung setzen und zwar zu anderen Phänomenen der Kommunikation. Musik hat nichts zu tun mit dem biologischen Prozess des Sex als körperlichem Akt der Fortpflanzung, allerdings sehr viel mit den kommunikativen Prozessen, die dem Geschlechtsverkehr vorausgehen (können): der Erotik. Dabei sind die kommunikativen Aufgaben und Funktionen dessen, was wir als »Musik« bezeichnen, andere als die des Balzgesangs eines Zebrafinken und viel komplexer als biologisch notwendig.

\section{Liebe, Erotik, Musik in der Kommunikation}

»Erotik«, »Liebe«, »Musik« sind drei hochkomplexe Begriffe mit extrem weiten Extensionen; alle drei sind mit ungeheuer vielen Bedeutungen aufgeladen. Uns interessiert hier nur ihre kommunikative Funktion als die eine Ebene, auf der wir sie alle drei vergleichen und miteinander in Beziehung setzen können. ${ }^{1}$

1 Eine Problematik besonders der Literatur aus musikwissenschaftlicher Perspektive ist die Tatsache, dass manche Autoren mit sehr unscharfen, alltagssprachlichen Begriffen operieren. Da wird z.B. Sex mit Erotik gleichgesetzt, Musik zum Sex mit erotischer Musik und diese mit Musik mit sexuell konnotierten Texten; Symbolen der Erotik in der Komposition werden psychologische Wirkungen zugeschrieben und jede Form von Körperlichkeit als Merkmal erotischer Sinnlich- 
Liebe hat in der Kommunikation die Funktion eines symbolisch generalisierten Kommunikationsmediums, das das Problem der hochpersönlichen Kommunikation löst, lautet die Kernthese Niklas Luhmanns in Liebe als Passion (1982: bes. 21-40). Kommunizierte Zeichen der Liebe machen es möglich, dass man von den intimsten Gedanken, Bedürfnissen, Gefühlen sprechen kann, ohne dass es peinlich oder gar völlig verrückt wirkt. An sich ist die Darstellung der eigenen Individualität, das Umkehren des Innersten nach außen, alles andere als kommunikativ. Wen interessieren schon die ganz individuellen Gefühle und Ansichten eines Fremden? Emotionale Exhibitionisten lösen befremdetes Schweigen oder gar schnellstmögliche Flucht aus, aber keine Kommunikation. Erst Signale der Liebe, direkt oder unterschwellig mitgeteilt, machen Kommunikation über das Ich möglich und wahrscheinlicher. Luhmann (1984: 220), der Medien als Phänomene auffasst, die das Zustandekommen von Kommunikation wahrscheinlicher machen, nennt Liebe daher ein symbolisch generalisiertes Medium, das anders als ein Verbreitungsmedium wie die Schrift oder ein Verstehensmedium wie die Sprache als Erfolgsmedium die Wahrscheinlichkeit erhöht, dass eine Mitteilung genau das Verhalten beim Gegenüber hervorruft, das beabsichtigt wurde: In diesem Fall die Toleranz und Aufmerksamkeit gegenüber hochindividueller Kommunikation (Luhmann 1982: 21-40). Wenn zwei jeweils nur von sich sprechen und doch Kommunikation weiterläuft, ist Liebe im Spiel.

Zwei, die sich gegenseitig ihre privatesten Gedanken und Gefühle mitteilen, konstruieren um sich herum eine Nahwelt, die sie als Paar auch gegenüber der Umwelt abschließt. Während Kommunikation immer auch Wahrnehmung und Erfahrung von Differenz bedeutet, durch Widerspruch und falsches Verstehen (Luhmann 1989: 136), führt die wechselseitige Akzeptanz an sich unmöglicher Kommunikation, das Hin- und Für-wahr-Nehmen des Sprechens vom Ich, zum Eindruck von Einigkeit, ja schließlich sogar

keit aufgefasst. Es ist mir bewusst, dass »Erotik«, »Liebe« und »Musik« im Alltag und in anderen wissenschaftlichen Disziplinen auch anderes und mehr bedeuten als Kommunikation. Doch wollte man Musik in einem wissenschaftlichen Kontext gleichzeitig auch als (Kunst-)Werke tönend bewegter Formen, auch als neurologisches, tiefenpsychologisches und verhaltensbiologisches Stimulanz, auch als akustisches Phänomen verstehen und Liebe sowie Erotik auch als Emotionen, auch als physiologische, psychologische biologische und kulturelle Phänomene, setzte man damit zwangsläufig Dinge gleich, die faktisch nicht gleich sind, sondern nur gleich scheinen, weil sie umgangssprachlich gleich bezeichnet werden. Die Diskussion der Gleichsetzung von Musik mit Balzverhalten in der sogenannten Biomusicology hat uns diese Problematik bereits deutlich gemacht. Eine methodisch saubere wissenschaftliche Beweisführung muss zunächst ein Tertium Comparationis benennen, das Gleiche, das es erlaubt »Erotik«, »Liebe « und »Musik« zu vergleichen. 
Einheit. Beide Kommunizierenden glauben, sich schließlich so gut zu kennen, dass sie ohne zu kommunizieren meinen, miteinander zu kommunizieren. Liebe bestätigt sich auch dadurch, dass beide dasselbe sagen, dass beide bei einem Song dasselbe zu fühlen meinen. Sexualität kann für diese Definition von Liebe zwar als Motivation im Hintergrund mitgedacht werden, sie ist jedoch nicht grundsätzlich notwendig, wie das Beispiel der Freundesliebe, der philia, zeigt (die Luhmann in seinem Ansatz ausblendet). Liebe gewinnt in ihrer Geschichte als Medium der Kommunikation ihre Dynamik gerade dadurch, dass sie mit komplexen Ritualen die unmittelbare Erfüllung körperlicher Triebe aufschiebt, in kulturellen Kontexten wie dem literarischen fin'amor der Trobadors und Minnesänger sogar bis in eine fantastische, unmögliche Zukunft.

Mein Begriff der Erotik im Kontext einer Analyse von Kommunikation lehnt sich an den der Liebe Luhmanns an, differenziert ihn jedoch weiter. Erotik lässt sich, so behaupte ich, der Liebe ähnlich, ebenfalls als ein symbolisch generalisiertes Medium beschreiben, das hochpersönliche Kommunikation ermöglicht. Zeichen der Erotik, die in die Kommunikation mit einfließen, ermöglichen die hochindividuelle bzw. intime Selbstdarstellung der Geschlechtlichkeit des Körpers. Der Unterschied zur Liebe liegt darin, wie Individualität dargestellt wird: Richtet sich Liebe auf die Darstellung dessen, was man umgangssprachlich gerne »innere Werte « nennt, auf das Fühlen und Meinen, die individuelle Weltsicht, richtet sich Erotik auf die Kommunikation individueller, körperlich geschlechtlicher Eigenheiten und Bedürfnisse. Statt des eigenen Denkens und Fühlens zeigt und entblößt man - zumindest symbolisch und ggf. auch verbal - den eigenen Körper, hebt Grenzen der Unberührbarkeit auf, öffnet sich, macht sich zugänglich. Man lenkt die Aufmerksamkeit des anderen auf die eigene körperliche Geschlechtlichkeit, die eigene Lust, und wenn er tatsächlich statt mit Abwendung mit Aufmerksamkeit reagiert, hat er die Chance, ebenfalls seine individuelle Körperlichkeit herauszustellen.

Auch eine solche äußerliche Selbstdarstellung gefährdet an sich Kommunikation. Sie ist nur dann in unserer Gesellschaft nicht peinlich, wenn sie als Erotik verstanden werden kann und mit Erotik oder doch zumindest mit deutlich erkennbarer Aufmerksamkeit beantwortet wird. Erotik, wie die Liebe auch, erzeugt dadurch, dass wechselseitig ein Verhalten toleriert wird, das eigentlich zu einem Abbruch von Kommunikation führen müsste, eine Nahwelt, die zwei Kommunizierende von der Umwelt absondert. Ziel ist auch hier das Paradoxon der Aufhebung der durch Kommunikation erzeugten Differenzen eben durch Kommunikation. Indem beide Kommunikationspartner ganz ihre individuelle Körperlichkeit, ihre eigenen Bedürfnisse in 
den Mittelpunkt der Kommunikation stellen und die Darstellung der Individualität des anderen akzeptieren und tolerieren, wird das Hin und Her, das Für und Wider der Kommunikation scheinbar aufgehoben. Jeder kann ganz bei sich selbst sein und gerade deshalb den Eindruck der Verschmelzung mit dem oder der anderen haben. Verbale Sprache, die für die Liebe notwendig ist, wird hier nicht benötigt. Dementsprechend lässt sich Erotik auch viel schneller herstellen als Liebe, die als ein Prozess des gegenseitigen Vertrautwerdens und -machens mit dem Denken und Fühlen ein langer, ja eigentlich endloser Prozess des gegenseitigen Akzeptierens und Verstehenwollens ist. Erotik ist jedoch auch viel flüchtiger. Sex, der biologische Akt des Geschlechtsverkehrs, ist mögliches Ziel und Motivation. Erotik macht das Zustandekommen von Sex wahrscheinlicher. Der tatsächliche Vollzug ist jedoch weder zwangsläufige Folge noch unbedingte Voraussetzung. Das Gefühl körperlicher Einheit braucht keine körperliche Vereinigung. Eine solche "Selbstverwirklichung « des einen mithilfe eines anderen Körpers kann Teil der Liebe sein, ist jedoch auch ohne sie möglich. ${ }^{2}$

Auch die Musik lässt sich, reduziert auf ihre Funktion in der Kommunikation, als symbolisch generalisiertes Kommunikationsmedium beschreiben. Sie regelt das Problem der gleichsinnigen Kommunikation (vgl. Helms 2009: 82). Wenn beide Seiten dasselbe wollen und tun und das auch wissen, ist Kommunikation eigentlich nicht notwendig. Das Einverständnis, das durch Liebe und Erotik mühsam, mit hohem Zeitverbrauch und dem ständigen Risiko des Abbruchs von Kommunikation hergestellt werden muss, erreicht Musik sofort. Nicht nur Paare, auch größere soziale Gruppen brauchen Mittel und Wege, sich aus sich selbst heraus ihrer selbst zu versichern, und genau das leistet Musik. Alle tun das gleiche, wollen das gleiche und weil alle Bescheid wissen, kann Kommunikation stark heruntergefahren werden auf die gegenseitige Kontrolle der Gleichsinnigkeit. Tänzer müssen darauf achten, im gleichen Rhythmus zu bleiben und dieselben ritualisierten Bewegungen zu machen. Musiker müssen ihren Vortrag, ihre individuelle Stimme oder ihr individuelles Spiel so an ihre Ensemblepartner anpassen, dass aus den Teilen ein sinnvolles Ganzes entsteht. Weitere Kommunikation ist nicht notwendig. So können sie ganz bei sich sein, ganz sie selbst, und doch ein

2 Auch die Darstellung von Macht basiert letztendlich auf der Herausstellung der individuellen Körperlichkeit. Wie der Sex im Fall der Erotik steht hier die Androhung körperlicher Gewalt im Hintergrund. Macht ist ein symbolisch generalisiertes Kommunikationsmedium, das mithilfe komplexer und subtiler Zeichen, die längst nicht mehr nur im Vorzeigen von Größe, Brustumfang und Armmuskulatur bestehen, körperliche Gewalt durch Androhung von Gewalt zu verhindern sucht. 
starkes Gemeinschaftsgefühl haben; so können Tanz und Musikmachen zu einem Rausch, zu einem Flow-Erlebnis oder gar zu einer Ekstase werden.

Das Beispiel der Tänzer in Trance zeigt, dass diese intern erzeugte Geschlossenheit zeitlich limitiert sein muss, damit das kurzgeschlossene System nicht zusammenbricht. Musik kommuniziert daher von Beginn an immer auch das Ende der Kommunikation mit. Durch ihre zeitliche Limitierung wird Musik zu einem Sonderfall der Kommunikation, die ja eigentlich als potentiell endloser Kreislauf definiert ist. Noch etwas unterscheidet die Musik als symbolisch generalisiertes Kommunikationsmedium. Während Zeichen der Liebe und Erotik in der Regel in Kommunikationssituationen eingesetzt werden, in denen sich zwei Individuen gegenüber stehen, werden Zeichen der Musik meist in Kommunikation unter vielen eingesetzt: alle mit allen, wenige mit vielen oder einer mit vielen. Musik hebt sich von der »gewöhnlichen « Kommunikation eines sozialen Systems ab: als Abschnitt mit eigenen Regeln und eigener Geschlossenheit. Sie ist damit dem Spiel vergleichbar, das ebenfalls eigene Regeln und einen im Vorhinein festgelegten Zeitverbrauch, jedoch wechselsinnige Kommunikation aufweist, ein Spiel, das alle Beteiligten gemeinsam spielen, dass jedoch auch (im Konzert) vorgespielt werden kann. Musik als symbolisch generalisiertes Kommunikationsmedium markiert eine Sonderwelt der Kommunikation, die sich vom Alltag abhebt.

Das Medium Musik hat viel mit den Medien Liebe und Erotik gemeinsam. Alle drei produzieren im Idealfall einen Status des Ganz-bei-sich-Seins in einer Gemeinschaft. Dabei wird nicht das Ich gelöscht und aufgehoben, sondern im Gegenteil: Es kann ganz Ich sein, ohne einen kommunikativen Widerpart, der fragt und nicht versteht. Die eigenen kommunikativen Handlungen finden nur Bestätigung vom Gegenüber, das im Idealfall anscheinend versteht ohne das Hin- und Her der Kommunikation. Das Ich potenziert sich zur Gemeinschaft und es entstehen Nahwelten, die sich von der Umwelt abschließen. Bei der Liebe geschieht dies durch das wechselseitige Kommunizieren von Gefühlen und Meinungen, das beiden Partnern den Eindruck von Vertrautheit und Nähe gibt. Ähnlich funktioniert die Erotik, bei der die riskante gegenseitige Kommunikation der individuellen Körperlichkeit die Umwelt ausschließt. Und ähnlich auch die Musik, bei der gleichsinnige Handlungen der Gruppe, das gemeinsame Tanzen, Singen oder Hören, einen engen Kreis um Musiker wie auch um Musiker und Hörer zieht.

Die drei Medien unterscheiden sich allerdings durch ihr Verhältnis zur Zeit. Die Liebe versucht, den Zustand der Nahwelt zeitlich unbegrenzt zu halten. Die Musik dagegen ist als Sonderwelt bzw. als Spiel mit eigenen Regeln notwendig zeitlich genau begrenzt. Die Erotik schließlich will unendlich und kann doch nur endlich sein. Man kann sie ähnlich einem Spiel ver- 
stehen - als zeitlich limitierte Sonderwelt - und auch als Teil der endlosen Vertrautheit und Vertrauensbildung der Liebe auffassen.

Nicht selten wird die Entstehung von Nahwelten in musikalischen Kommunikationssystemen von Beobachtern bewusst oder unbewusst nicht auf das Medium der Musik, sondern auf Liebe und Erotik zurückgeführt; eine durchaus produktive Verwechslung, mit deren Hilfe die Musik Brücken bauen kann: Ein gleicher Musikgeschmack ist für viele Paare wichtiges Zeichen des gegenseitigen Einverständnisses (Gembris 1995: 124f.). Eine besondere körperliche Harmonie beim Tanzen kann durchaus auch in Erotik übergehen. Die Nahwelt, die zwei Musizierende umgibt, kann - wie in Lew Tolstois Novelle Kreutzersonate dargestellt - Zuhörer zur Eifersucht treiben. Musikalische Synchronisation lässt die Synchronisation von Geist und Körper vermuten (Kreutz 1997: 298). Doch zeigt gerade das Beispiel des rasenden Ehemanns der Kreutzersonate die Besonderheit des Mediums Musik, die eine Gleichsetzung mit Liebe oder Erotik gefährlich macht. Durch die Produktion einer Sonderwelt, in der musikalische Kommunikation abläuft, durch ihren Spielcharakter, bleibt unklar, ob das Kommunizierte eigentlich oder uneigentlich gemeint ist. Ein »| love you « in einer unmittelbaren Face-to-FaceSituation geäußert, aber gesungen, hinterlässt den Zweifel, ob dieses Liebesgeständnis Spiel oder Realität sei. Das ist das große Risiko, aber auch die große Chance musikalischer Codes: Denn Musik kann durch die Sonderwelt, die sie schafft, in der Alltagswelt tabuisierte oder unmögliche Kommunikation möglich machen. So können Handlungen des Flirtens und der Anmache, die Sprache der Liebe und die Zeichen der Erotik unverbindlich erprobt, vermittelt und zur Diskussion gestellt werden. Gerade in Kulturen, die auf dem Prinzip der Kontrolle körperlicher Triebe durch ritualisierte Kommunikation von Körperlichkeit basieren, ist Musik daher sinnvoll und notwendig.

Der uneigentliche Charakter der Musik macht sie - um noch einmal auf Darwin zurück zu kommen - denkbar ungeeignet für die »Balz«. Wer um einen Partner werben will, muss ihr oder ihm deutlich machen, dass es ernst gemeint ist. Ein Spiel taugt dazu nicht. Die Erotik zwischen Musikern und Publikum und selbst zwischen zwei Tänzern steht unter Fiktionsvorbehalt und muss, wenn die Musik endet, eine spürbare Hürde überwinden, um in die Realität hinübergetragen zu werden. Zudem ist es ja nicht die Musik, die lockt und wirbt, wie die Lautäußerungen der Tiere. Musik stellt lediglich den kommunikativen Rahmen her, in dem - unverbindlich und spielerisch um einen Sexualpartner geworben werden kann. 


\section{Soziale Sonderräume der Erotik}

Je enger Menschen zusammenleben und je größer und heterogener die Gruppen sind, die zusammen leben und kommunizieren müssen, desto komplexer wird das Verhalten, das der Kontrolle bzw. der Verzögerung oder Umlenkung des spontanen affektiven Verhaltens dient. Um das Ausleben von Gewalt und unerwünschtem Sex, der die Paarbindung gefährden könnte, zu unterbinden, führen Gesellschaften komplexe Riten und Regeln ein, die den spontanen Ausbruch von Körperlichkeit unwahrscheinlich machen. Hierarchisch strukturierte Gesellschaften erfinden hochkomplexe Zeichensysteme der Macht, die die Ausübung von Gewalt zur Durchsetzung des Willens unnötig machen. Ähnlich komplex werden auch die Zeichensysteme der Erotik, je komplexer eine Gesellschaft wird. Ob man dieses Anwachsen von Komplexität evolutionär verstehen kann wie der Soziologe Norbert Elias, der hierfür den Begriff des »Prozesses der Zivilisation« prägte (Elias 1997), sei dahin gestellt (vgl. u.a. die Kritik in Duerr 1995 und 1999). Sicher ist, dass Kommunikation, die einen gewissen Grad an Komplexität überschreitet, an Eindeutigkeit verliert und schließlich Gefahr läuft, an Verstehensproblemen zu scheitern.

Ohne Erotik, die gegenseitige Präsentation und Akzeptanz der individuellen Geschlechtlichkeit des Körpers, kann körperliche Kommunikation zur Ermöglichung von Sex auch und nur noch als Demonstration von Macht mit der Androhung von Gewalt ablaufen. Eine radikale Tabuisierung erotischen Verhaltens gefährdet daher nachhaltig die sexuelle Selbstbestimmung der Geschlechter einer Gesellschaft. Eine Tabuisierung auch von Gewalt zwischen den Geschlechtern müsste schließlich - biologisch gesprochen zu Problemen mit der Erhaltung der Art führen. Viele Gesellschaften mit entsprechenden Tabus begegnen diesem Problem durch Differenzierungen von Öffentlichkeit und Privatheit, markiert durch das Haus und die Ehe (vgl. Elias 1997: 351-356), und damit der Schaffung einer geschlossenen Sphäre, die die moralischen Instanzen der Allgemeinheit weniger streng kontrollieren. $^{3}$

Eine zunehmend komplexer werdende Gesellschaft braucht allerdings aus verschiedenen Gründen auch Reservate der Körperlichkeit außerhalb der

3 Diese Trennung hatte und hat oft fatale Folgen für Frauen, die gezwungen werden, jede Öffentlichkeit zu vermeiden, um nicht in den Verdacht zu geraten, öffentlich zu sein - das galt auch und gilt auch noch heute besonders für Schauspielerinnen und Musikerinnen. Die Situation der Frau in der Musik ist mittlerweile gut, wenn auch noch nicht erschöpfend erforscht und soll an dieser Stelle nicht weiter verfolgt werden. 
Privatheit des Hauses: um Triebabfuhr zu ermöglichen und den Druck von den Grenzen der Tabus körperlicher Kommunikation zu nehmen (durch Sport z.B.); um die Normen und Tabus in Frage stellen zu können, sie zu verschärfen oder aufzulockern, damit sie sich an geänderte Umwelten anpassen können; und schließlich auch, um den Mitgliedern der Gesellschaft die Chance zu geben, körperliche Kommunikation zu lernen. Musik trägt in vielen Fällen dazu bei, solche Sonderwelten zu markieren. Sie dient auch der Repräsentation der Macht, ist aber vor allem wichtig für die Schaffung und Abgrenzung von Reservaten der Erotik. So beobachtet Gunter Kreutz (1997: 297): »Musik gibt es überall dort, wo Individuen untereinander - zum großen Teil imaginierte - Beziehungen eingehen.«

In der griechischen Antike stand - und steht vermittelt über die Bibel (Offenbarung des Johannes, z.B. Kap. 17, 5) bis heute - die »Hure Babylon« als Symbol für sexuelle Verderbtheit. In der Tat überliefern Keilschrifttafeln der frühesten Hochkulturen in Mesopotamien einen großen Korpus wunderbarer erotischer Dichtung und einen Götterhimmel, in dem die Göttin für Sexualität, sumerisch Inana, babylonisch Ištar, eine hohe Bedeutung hatte. ${ }^{4}$ Sammlungen von Omen, die einige sexuelle Praktiken mit Flüchen belegen und andere mit Glücksverheißungen verknüpfen, zeigen, dass es in den Keilschriftkulturen am Ende des 2. und dem Beginn des 1. Jahrtausends vor Christus auch um eine soziale Kontrolle der Triebe gegangen sein könnte. Die Riten der Kulte der Liebesgöttin können auch als sozial kontrollierte Triebabfuhr gedeutet werden. Ihre Kultdiener - meist Sklaven und von geringem sozialen Ansehen - waren Ekstatiker, die sich durch Gesänge und Tänze in Trance versetzten. Sänger mit Harfen und Flöten, aber auch Kultdiener mit Prügeln und Riemen brachten die Göttin zur Ruhe (Haas 1999: 71f.). Motiviert und legitimiert durch die Religion und als Fest markiert durch den Ort des Tempels und die Musik, sind hier die Elemente des Außersich-Geratens, der Ekstase, vereint, die eine komplexe Gesellschaft im Alltag gefährden können: Erotik und Gewalt. Im Tempel scheint ein legitimer Ort für Verhalten gewesen zu sein, das außerhalb problematisch war. Hier brachte homosexueller Verkehr mit Tempeldienern Glück, während außerhalb Sex mit Niederrangigen Unglück verhieß (ebd.: 21). Die assinū-Tempeldiener trugen Frauenkleider, sangen in der sumerischen »Frauensprache « und wurden manchmal als Dienerinnen bezeichnet (ebd.: 70). Ihre Inter-

4 Eine sumerische Quelle beschreibt sie als Göttin für die Geschlechtsteile von Mann und Frau, verschiedene Sexualpraktiken, die Prostitution, aber auch für das Diffamieren, Verleumden und Schmeicheln, eine Göttin also mit unberechenbarem, anarchischen Wesen (Haas 1999: 18, 30-33). 
sexualität, ihr musikalisch-erotisches Außer-sich-Sein, machte sie zu Mittlern zwischen Götterwelt und Menschen (ebd.: 73).

Das antike Griechenland kannte mit Dionysus einen Gott, dessen Zuständigkeit man pauschalisierend mit »Grenzüberschreitungen« beschreiben kann: zwischen Nüchternheit und Rausch, zwischen Anstand und Unmoral, zwischen Mann und Frau, zwischen Stadt und Land (Schlesier/Ley 2010). Aus dem Dithyrambos, dem Kultlied des Dionysus, soll das Theater hervorgegangen sein, und so repräsentiert er vielleicht auch die Grenze zwischen Realität und Fiktion menschlichen Verhaltens. Vasenbilder ordnen der Sphäre des Dionysus-Kults vor allem Schlaginstrumente und den Aulos zu, ein Doppelrohrblattinstrument mit zwei Rohren, dem ekstatische Wirkung zugeschrieben wurde (Aristoteles 2005: 58, Politika 1341a20-25). Livius schildert in römischer Zeit die dem Dionysus geweihten Bacchanalien als Orte der Ausschweifung, die zu einem Skandal wurden, weil sie sich zunehmend öffneten (Frateantonio 2010), d.h. als Sonderraum in den öffentlichen Raum vordrangen.

Symposien, die griechischen Gastmähler, an denen nur Männer teilnahmen, gingen, glaubt man den Quellen, vermutlich oft in einen vom Symposiarches systematisch gesteuerten Alkoholrausch über, der, wie Malereien auf Vasen und Trinkgefäßen vermuten lassen, in sexuellen Ausschweifungen zur Musik enden konnte. Während die (Ehe-)Frauen des Hauses öffentlich nicht in Erscheinung treten durften, wurden in dieser Phase des Symposions neben Lustknaben auch Hetären als Musikerinnen und Liebesdienerinnen zugelassen (vgl. Guerrieri/Giustozzi 2007: 40-43).

Mit dem Siegeszug des christlichen Glaubens wurde die erotische Ekstase nach und nach aus dem religiösen Kult verdrängt. Mit der Festlegung (auf dem Konzil von Benevent 1019) einer 40tägigen Zeit vor Ostern, in der Buße durch körperlichen Verzicht Pflicht war, prägte sich vor dem Beginn dieser Bußzeit die Fastnacht bzw. der Karneval aus. Diese Zeit der lizenzierten Ausschweifungen wird bis heute von spezieller Musik begleitet, von mündlich überlieferter Vokalmusik und instrumentalen Bräuchen über die mehrstimmigen Canti carnescialeschi in Florenz am Ende des 15. Jahrhunderts bis hin zur Oper, die im italienischen Karneval Mittelpunkt der Feiern war, und dem Karnevalsschlager der Gegenwart.

In vielen Kulturen gehörten musikalische Fähigkeiten zu den beruflichen Qualifikationen von Prostituierten (s. die Beiträge in Feldman 2006), von den griechischen Hetären über die Kurtisanen der frühen Neuzeit bis zu den Tänzerinnen der Nachtclubs der Gegenwart. Wenn sie nicht selbst musizieren, so tanzen sie für ihr Publikum und ihre Freier, simulieren öffentlich privates, intimes Verhalten. 
Im Tanz wird ganz bewusst und sehr direkt die Aufmerksamkeit des Betrachters auf den Körper der Tanzenden gelenkt. Die vorgeführte Musik, jedes Spiel vor Publikum, hebt Regeln der alltäglichen Kommunikation auf und erlaubt den Zuschauern das Hinsehen ohne Scham. Tanz und Vorspiel allein laden schon zur »voluptas oculorum [...] carnis« (Augustinus 1981: 182, Confessiones 10, 34,51), zur Fleischeslust der Augen der Betrachter, ein, auch ohne explizite Erotik in den Handlungen derjenigen, die sich betrachten lassen.

Die schamlose Beachtung und Betrachtung von Körpern in der Öffentlichkeit wird für die Dauer der Musik legitimiert, wenn auch nur als Sonderwelt, für die es einen Preis zu zahlen gibt, der dem Publikum zusätzlich deutlich macht, dass es sich hier um ein - wenn auch oft sehr ernstes Spiel und nicht um wirkliche Anmache handelt. Dass die Musik hierbei für manche Zuschauer ein Realitätsproblem erzeugt, belegen nicht nur Striptease-Bars, in denen darauf geachtet wird, dass die Grenze zwischen Bühne und Zuschauerraum auch eine Grenze zwischen optischer Erotik und handfester Sexualität markiert. So steigen mit der Erotik einer Aufführung, mit ihrer scheinbaren »Echtheit «, auch die Preise, die für die Annäherung an die Realität zu zahlen sind, um die Realität wiederum auf Abstand zu halten und den Spielcharakter schmerzhaft deutlich in Erinnerung zu rufen. Die biblische Salome konnte so von ihrem Stiefvater ganz gegen dessen ursprünglichen Willen als Preis für ihren Tanz den Kopf des Johannes fordern (Markus 6, 23). Noch teurer bezahlte im hetitischen Mythos das Meeresungeheuer Hedammu. Das Mädchen Šawoška und ihre Dienerinnen versuchen, das unersättliche Schlangenwesen aus dem Wasser zu locken, damit es getötet werden kann. Die Musik von Becken und Trommeln und der Tanz der nackten Frauen zeigen ihre Wirkung auf das Ungeheuer: »seine Männlichkeit springt immer wieder hervor «, schreiben die alten Keilschriftquellen. Tanz und Musik ermöglichen den Frauen glaubhaft, ihre Körper zu präsentieren - und dem Monster unterläuft ein fataler Fehler: Es setzt Tanzbewegungen, musikalisch begründete Selbstdarstellung, mit sexueller Anmache gleich, wagt sich hervor und wird getötet (Schuol 2004: 209). Es ist riskant, das Verhalten in musikalischen Sonderräumen mit der Alltagskommunikation zu verwechseln.

Musik markiert als Medium Sonderwelten, in denen Verhalten möglich ist, das außerhalb tabuisiert ist. In der langen Geschichte des menschlichen Verhaltens der Feier - vom religiösen Ritus bis zur privaten Party - zeigt die Musik an, dass andere Regeln der Kommunikation gelten als im Alltag. Bei vielen Tänzen sind Anfassen und körperliche Nähe erlaubt, weil Berührungen hier durch den Tanz begründet sind und daher nicht dieselbe 
Bedeutung haben wie in der Alltagskommunikation. Damit stellen musikalische Sonderwelten aber auch eine ständige Provokation der Alltagskommunikation dar und fordern die Gesellschaft mit Fiktionen einer direkten Körperlichkeit heraus.

Musikalische Riten grenzen den Alltag und die Umwelt aus, schaffen aber auch innerhalb der durch sie erzeugten Nahwelt einen Zusammenhalt. Die gemeinsame, ritualisierte Grenzüberschreitung der antiken Symposien war in der griechischen Kultur ein wichtiges Mittel, politische und soziale Koalitionen zu festigen. Das gemeinsame Singen liederlicher Lieder auf dem Herrenabend oder in Institutionen wie den englischen Catch and Glee Clubs des 17. und 18. Jahrhunderts, das kollektive Hören von Tonträgern mit Titeln wie Ohne Hemd und ohne Höschen, wie sie z.B. Polydor in den 1970er Jahren in langer Serie herausgab, oder von CDs eines Mickie Krause produziert nicht nur durch die Musik ein Gefühl von Zusammenhalt, sondern auch durch die gemeinsam begangene Devianz, den kollektiven Tabubruch, der leicht fällt und wenig riskant ist, da er in einer Sonderwelt stattfindet. Das abweichende Verhalten kann Fiktion bleiben, ein Spiel, ein So-tun-alsob. Dabei ist die solidarisierende Wirkung nach innen umso stärker, je provozierender das Verhalten nach außen wirkt. Die Verletzung von Tabus hilft zu differenzieren: Den Musiker, der noch einen Schritt weiter geht, von seinen Konkurrenten; den Hörer, der sich ansprechen lässt, von denen, die sich schockiert abwenden.

Trotz ihrer Nicht-Alltäglichkeit haben Feiern und musikalische Sonderwelten Konsequenzen für den Alltag. Ihre Besonderheit ist gerade das Changieren zwischen Fiktion und Realität. Rituelle religiöse Feiern werden abgehalten, um den Kontakt zu den Göttern herzustellen, damit diese um Unterstützung im Alltag gebeten werden können. Religiöse und weltliche Feiern werden abgehalten, um soziale Gemeinschaften für den Alltag herzustellen und zu festigen, was besonders dann erfolgreich ist, wenn die Umwelt durch geheime Riten bzw. Insiderwissen oder durch gezielte Provokation auf Distanz gehalten wird. Das funktioniert auch bei den kommerziellen Feiern der Neuzeit mit einem zufälligen, nicht geladenen, anonymen Publikum: in der Diskothek z.B. Eine Verlängerung der entstandenen Gemeinschaft der Tänzer über das Ende der Feier hinaus ist hier allerdings deutlich unwahrscheinlicher.

Auf dem Fest kann die Entstehung körperlicher Nahwelten erprobt, Erotik beobachtet, nachgemacht und gelernt werden. Das alles ist unverbindlich, mit einem Fiktionsvorbehalt. Solange die Musik spielt, kann man auch so tun als ob. Dementsprechend existiert trotz der Möglichkeit körperlicher Selbstdarstellung immer auch noch eine Schwelle zur tatsächlichen, 
ernst gemeinten Erotik. Die Lizenz zur unverbindlichen Selbstdarstellung des Körpers gibt es nur um den Preis der Unsicherheit zwischen Spiel und Ernst, um das Tabu der Alltagskommunikation nicht zu gefährden.

Viele Gesellschaften achteten und achten streng auf die Differenz zwischen Alltag und Fest, nur in der so genannten »westlichen Welt « beginnen die Sonderwelten in den Alltag hinüberzuwuchern, doch dazu unten mehr. Feste werden begrenzt, durch das Ende von Feiertagen, durch Emissionsordnungen akustisch von den Nachbarn getrennt, durch Öffnungszeiten von Gastwirtschaften limitiert. Institutionen werden ausgebildet, um über diese Grenzen zu wachen. So stehen sich Moralwächter mit ihren Institutionen und Medien und Tabubrecher mit ihren Institutionen und Medien gegenüber - nicht selten je nach Saison und Gelegenheit durchaus auch in personaler oder medialer Union. Der »Prozess der Zivilisation«, will man diesen Begriff weiterhin verwenden, macht keine besseren, zivilisierteren Menschen. Er macht nur Verhalten, das zu Sex oder körperlicher Gewalt führen kann, komplexer und schwieriger und zögert damit beides heraus bzw. macht das Zustandekommen unwahrscheinlicher. Er gewinnt seine Energie aus einer Differenz zwischen den körperlichen, individuellen und egoistischen Bedürfnissen nach maximaler Fortpflanzung und Durchsetzung eigener Überlebensvorteile, die eine Gesellschaft gefährden, und den sozialen Bedürfnissen von Gleichheit, körperlicher Unversehrtheit, Verständnis, Altruismus bzw. Rückstellung der individuellen Bedürfnisse im Interesse der Gemeinschaft, die das Überleben des Individuums gefährden. So entsteht aus diesem unlösbaren Widerspruch ein Motor, der, ständig kreisend, sich immer wieder selbst erschafft und - Luhmann würde sagen: »autopoietisch« - aus sich selbst heraus Kommunikation aufrecht erhält. Welch eine Chance für die Musik: Zwar produziert sie zunächst einmal Gemeinsamkeit, eine Nahwelt, indem sie alle, die nicht Musik machen und nicht zuhören wollen, aussperrt. Doch innerhalb dieses Nahbereichs kann sie Kommunikation weiter differenzieren: Sie kann Musiker von Zuhörern, Tänzer von Zuschauern, Komponisten von Interpreten als funktionale Rollen in der Kommunikation unterscheiden und damit auch Möglichkeiten für Individuen schaffen, sich aus der Gruppe herauszuheben. In ihrer nicht-alltäglichen Welt erlaubt die Musik das öffentliche Singen vom Ich und die öffentliche Darstellung der individuellen Körperlichkeit beim Tanz, die ein Ich gegenüber anderen Ichs innerhalb einer von der Umwelt abgeschlossenen Nahwelt positioniert. Aus dieser Differenz speist sich die Dynamik unserer Musikgeschichte wie auch die enge Verbindung der Musik mit Liebe und Erotik als symbolisch generalisierten Medien. Alle drei begleiten den gesellschaftlichen Prozess der Ausbildung immer längerer und immer stärker verflochtener Ketten sozialer Interdependen- 
zen, wie Elias (1997) schreiben würde, die sie allerdings durch eine ebenso zunehmende Individualisierung ausbalancieren.

\section{Individualität des Körpers gegen Individualität des Geistes}

Musik, wenn sie nicht in der Gruppe von allen für alle produziert, sondern vorgemacht, vorgetanzt oder als Noten vorgelegt wird, lenkt die Aufmerksamkeit derer, die sich auf sie einlassen, auf ihren Macher, auf seinen Körper oder seinen Intellekt. Vorgeführte Musik - und das gilt durchaus auch für Musik, die anderen von einer Schallaufzeichnung vorgespielt wird trägt zur Individuation des Vorführenden bei. Er oder sie erregt Aufmerksamkeit als eine Quelle musikalischer Informationen und hebt sich von anderen und von der Umwelt ab. Ein großer Teil der musikalischen Mitteilung bezieht sich zurück auf den Musiker, d.h. sie informiert das Publikum weniger über dessen mögliche Anschlusshandlungen - es hat nur die Wahl zwischen Zuhören oder Abwenden und muss lediglich mit Aufmerksamkeit reagieren - , sondern über Anschlüsse des Musikers: Hört her, schaut her, was ich kann, was ich ausdrücke, was ich fühle bzw. was ich habe, was mir gefällt. Die meisten Menschen wissen, dass solche intimen Mitteilungen im Alltag eigentlich hochriskant sind und in der Regel zum Abbruch von Kommunikation führen. Sie empfinden Hemmungen, Hemmungen, die sie mit den meisten Musikern teilen, die vor jedem Konzert das Gefühl haben, eine Schwelle überschreiten zu müssen. Auch das Vorsingen und Vorspielen ist eine Provokation des sozialen Systems. Es degradiert einen Großteil der Kommunikanden zu passiven Hörern, zwingt sie, ihre Aufmerksamkeit auf die Individualität des Musikers zu richten - eine doppelte Provokation besonders in hierarchischen Gesellschaften, wenn der Musiker vor Höhergestellten auftritt und diese zu einem Verhalten zwingt, das sonst nur Untergebenen zugemutet wird bzw. im Tanz sogar die Bewegungen seiner Herren steuert (Helms 2009: 84-87). ${ }^{5}$

Ganz besonders groß wird die Provokation, wenn der Sänger nicht nur wie z.B. die Barden und Spielleute des frühen Mittelalters - Geschichte(n) vorträgt, von mythologischen Helden oder noch lebenden Herrschern, son-

5 In vielen Gesellschaften wird diese Provokation dadurch gemildert, dass diese Darsteller, zu denen man auch Zelebranten religiöser Riten zählten kann, einen außergesellschaftlichen und -weltlichen Status haben, symbolisiert z.B. im Bereich des religiösen Kults durch körperlichen Verzicht (z.B. auf Nahrung oder Sex) oder, wie im Fall der mittelalterlichen Spielleute, durch die Ehr- und Rechtlosigkeit. 
dern ein »|ch« und dessen innerste Gefühle zum Thema seiner Lieder macht. Die Chansons der Trobadors und ihrer Nachfolger, der Trouvères und Minnesänger, müssen für die feudale Gesellschaft des Hochmittelalters eine außerordentlich reizvolle Provokation dargestellt haben. Dieses Singen vom Ich braucht eine gute Begründung, die vom Publikum akzeptiert werden muss, um überhaupt zu funktionieren, und eine solche Begründung liefert die Liebe. Der Sänger singt von Gefühlen für eine geheim oder doch anonym bleibende Angebetete, die sich die Hörer durchaus auch als im Publikum sitzend vorstellen können. Er macht seine Zuhörer zu Mitwissern und damit - für die Dauer des Songs und durch das Einverständnis, den intimen Geständnissen zuzuhören - in der Sonderwelt der Musik zu Freunden, wenn nicht sogar, indem er in seinen Texten ein »Du« anspricht, zu Geliebten. Bis in die Gegenwart handeln nicht-narrative Lieder, die vom Ich singen, in einem hohen Prozentsatz von der Liebe oder vom Begehren. Erst im HipHop der Gegenwart ist es möglich geworden, auch das Streben nach Macht, den Herrschaftswillen über andere, zur legitimen Begründung der Ich-Perspektive eines Liedes zu etablieren (vgl. ebd.: 93f.).

In der Geschichte der europäischen Musik wurde ihre soziale Sprengkraft des Vorführens und Vormachens auch dadurch entschärft, dass die Darstellung körperlicher Individualität in der Musik als niederrangig oder gar als gefährlich für Sitte und Moral markiert, während die Darstellung einer geistigen Individualität, die man nur mit einer gewissen Bildung erkennen kann, legitimiert wurde. Das, was nach der Erfindung der Notation und bis zur Erfindung der Schallaufzeichnung als Außenseite des Musikmachens wahrgenommen wurde, das, was man als Hörer tatsächlich wahrnahm, wurde abgewertet, die nicht wahrnehmbare intellektuelle Tätigkeit im Hintergrund dagegen nach und nach zum eigentlichen Kern der Musik erhoben. Seit der Antike ist in der Musikgeschichte eine Diskussion um die Provokation der Darstellung von Individualität durch Musik zu beobachten, die als Auseinandersetzung zwischen Ratio und Körper geführt wird. ${ }^{6}$

Im 5. vorchristlichen Jahrhundert ist zum ersten Mal der Konflikt zwischen Vorstellungen von einer minderwertigen sinnlichen, orgiastischen und

6 Musikgeschichten beschreiben allerdings bis heute fast ausschließlich die eine Seite: die der Ratio. Sie beginnen dort, wo Musikmachen als intellektueller Prozess begriffen werden kann: meist mit der Erfindung der Notation, die Musik als Materialisierung des Denkens eines individuellen, namentlich bekannten Komponisten unabhängig von den körperlichen Handlungen der Musiker machte. Die Musik des Körpers, das Musik-Machen, war allerdings vor der Erfindung der Schallaufzeichnung auch tatsächlich nicht geschichtsfähig. Sie war eine Musik des flüchtigen Augenblicks. Was wir als Historiker meist nur beobachten können, ist die Auseinandersetzung zweier Prinzipien: der geistigen Individualität mit der körperlichen Individualität. 
einer hochwertigen, eher rationalen Musik festzustellen. Zunächst erscheint er metaphorisch in der griechischen Sage vom Wettstreit des Marsyas mit Apoll. ${ }^{7}$ Die Philosophie greift diesen Konflikt zu Beginn des 4. vorchristlichen Jahrhunderts auf. Platon nimmt an, dass Musik menschliche Charakterzüge nachahme und dass die menschliche Seele durch Nachahmung diese Charaktere annehmen könne. Als Konsequenz hieraus fordert er, aus einer idealen Stadt alle weichliche, sinnliche, nach seinen Worten »weibliche « Musik zu verbannen (Platon 1971: 217-235, Politeia 398a-403d). Wohin solche Musik führt, wissen die Griechen aus der homerischen Odyssee und ihrem Bericht von Odysseus' Begegnung mit den Sirenen. Platon erlaubt nur noch kriegerische Modi und solche, die der Muße des Kriegers dienen. Die moderne Instrumentalmusik verdammt er als »voller Roheit«, ihnen fehle der »logos«, der Text, der bewirke, dass Musik nicht nur erregt, sondern auch eine rational erkennbare Bedeutung bekommt (Platon 1994: 56, Nomoi 669e-670b). Auch Aristoteles beurteilt diese Musik als eines freien Mannes nicht würdig, es sei eine Musik für Sklaven. Er weist ihr aber immerhin eine gesellschaftliche Funktion mit der heilenden Wirkung durch Ekstase, der Katharsis, zu (Aristoteles 2005: 57-59, Politika 1341a5-1341b20). Platon und Aristoteles sind sich einig, dass das wichtigste Erziehungsziel die Schulung der Urteilsfähigkeit über Musik ist - und zwar eine analytische Urteilsfähigkeit, die den ethischen Gehalt eines Stücks bewusst macht und erkennt. Die griechischen Philosophen konstruieren als erste den Kontrast zwischen einer angemessenen, rational zu durchdringenden, ethisch und ästhetisch hochstehenden Musik, die es zu bewahren gilt, und einer ekstatisch auf den Körper wirkenden, körperlichen, technisch virtuosen, aber sozial niederen Musik.

Die Kirchenväter übermittelten diese Differenz in eigener, christlicher Interpretation in das Mittelalter. Die frühen Christen verstehen jede öffentliche Präsentation von Individualität - des Geistes wie auch des Körpers als problematisch. Das öffentliche Auftreten von Rednern, Dichtern,

7 Der Silen Marsyas, als niederrangiger Quellgott bereits einer sinnlichen Sphäre zugeordnet, lernt virtuos das Doppelrohrblattinstrument Aulos spielen, das er zu eigener Erfindung nicht fähig - im Sumpf findet, wo es die Göttin Athene weggeworfen hat. Er fordert den Lyra spielenden Apoll heraus, den Gott der Harmonie - ein Begriff, hinter dem das philosophische Konzept einer zahlenmäßig proportionalen Ordnung steht. Die Musen, Töchter der Göttin des Gedächtnisses, Mnemosyne, sprechen Apoll den Sieg zu, weil er im Gegensatz zum Bläser Marsyas zu seinem Instrumentenspiel singen kann, und seiner Musik damit Sinn und Bedeutung gibt. Sie bevorzugen - wen wundert es bei der Mutter - die erinnerungswürdige Musik mit Text vor dem körperlich-virtuosen, ekstatischen Spiel des Augenblicks. Marsyas, der so sehr die Fähigkeiten seines Körpers, seine Fingerfertigkeit, ausstellte, wird mit der totalen körperlichen Entblößung bestraft: Er wird gehäutet. 
Schauspielern, Artisten und Musikern wird als Werk des Teufels verdammt, als Anreiz zu Ehebruch, Leichtsinn, Sinnlichkeit (Hartung 1982: 33). Es lenkt ab von der Auseinandersetzung mit dem Wort Gottes. Augustinus (1981: 181f., Confessiones 10, 33,49-50) betrachtet selbst die Musik im Gottesdienst mit Argwohn: Führt sie den geistig Schwachen zum Wort Gottes, ist sie gut, führt sie jedoch zu sinnlichem Genuss und wird quasi zum musikalischen Selbstzweck, sollte sie verboten werden.

In die mittelalterliche klerikale Literatur werden diese Ansichten eins zu eins übernommen. In seiner bekannten Beschreibung der Spielleute zählt Thomas von Aquin in der Mitte des 13. Jahrhunderts unter denen, die sicher der Verdammnis anheimfallen, solche auf, die ihre Körper in der Öffentlichkeit entblößen und unsittliche Tänze und Gesten aufführen, und solche, die Instrumente spielen und zur Erbauung der Menschen auf öffentlichen Trinkgelagen und ausschweifenden Versammlungen Lieder singen, die die Menschen zur Zügellosigkeit verführen. Nur Spielleute, die die Taten der Fürsten und die Viten der Heiligen besängen, die keine Unsittlichkeiten begingen und keine Tänze aufführten, die also anderen dienen und nicht sich selber darstellen, hätten eine Chance auf das Seelenheil (Hartung 1982: 38). Auf der einen Seite haben wir hier wieder die wertvolle, körperlose und Geschichte tradierende Musik - auf der anderen Seite die verdammenswerte, niedere Musik, die Körperlichkeit öffentlich ausstellt.

Kritisch wird jedoch nicht nur die Darstellung von Körperlichkeit gesehen, sondern auch die Entwicklung einer komplexen mehrstimmigen Musik, die für die Erfindungsgabe eines Individuums steht. Die Erfindung einer Notenschrift im 9. Jahrhundert setzt eine Entwicklung in Gang, die die Musik zunehmend unabhängig vom liturgischen Text macht. Geschriebene Musik erlaubt neue Komplexität: Mehrstimmigkeit zum Beispiel und Text-unabhängige Rhythmisierung. Sie lässt jedoch auch den Komponisten entstehen, der sein selbsterdachtes Stück zu einem fixierten Werk machen kann, das unabhängig von einer Gemeinschaft existieren kann, die in oralen Kulturen ein Stück tradiert und damit immer wieder neu schafft. Schnell kommt die Frage auf, ob die neue geschriebene Musik, kunstvoll und komplex, nicht eher zur Befriedigung der Selbstdarstellung des Komponisten dient und als unkeusche Eitelkeit zu verstehen ist. So schimpft im 12. Jahrhundert Johannes von Salisbury über eine neu komponierte Musik, die so komplex sei, dass der Hörer ihre Struktur nicht mehr erkennen könne. Sie diene statt dem Lobe Gottes den Musikern nur zur »Zurschaustellung ihrer selbst «, sei sinnlich, ja weibisch. Der Geist, vom Liebreiz der Musik umschmeichelt, könne die Bedeutung des Gehörten nicht mehr ausreichend prüfen, sodass sie durch Abweichen vom rechten Maß »schneller Geilheit der Lenden als Hingabe des 
Geistes« erregt (Übers. v. Verf. nach Johannes von Salisbury 1965, Bd. 1: 41). Nicht umsonst schreibt noch im 18. Jahrhundert Johann Sebastian Bach »SDG « - soli deo gloria - unter seine religiösen Kompositionen, wohl auch, um den Eindruck der Eitelkeit zu zerstreuen angesichts eines hochindividuellen Werkes.

Eine überdeutliche Kunstfertigkeit verweist für kirchliche Kritiker auf die Individualität des Komponisten statt auf Gott und ist daher genauso unsittlich und unkeusch, wie die öffentliche Präsentation des Körpers. Dennoch ist die Entwicklung der kunstvollen Musik zur Kunstmusik nicht aufzuhalten. Mit der Notenschrift setzt sich der Komponist nach und nach als ein dem Schöpfer vergleichbares Individuum durch. Der Siegeszug der weltlichen Musik nach Reformation und Gegenreformation führt zu einer Veränderung der Werte: Immer mehr rückt die geistige Originalität bzw. Individualität des Komponisten, repräsentiert in der Komposition, in den Mittelpunkt der Betrachtung. Sie wird, mit der notwendigen Demut betrieben, zur guten Seite der Macht der Musik, die sich ständig der negativen, körperlich-sinnlichen Seite erwehren muss. Besonders musikpädagogische Traktate diskutieren diese Differenz seit der Renaissance intensiv.

Der entscheidende Schritt für die Trennung der körperlichen von der geistigen Seite der Musik ist jedoch die Ausdifferenzierung der musikalischen Ästhetik im 16. und 17. Jahrhundert. Aristoteles beschreibt in Über den Himmel, dass Geräusche und Töne durch Bewegung von Körpern entstehen (Aristoteles 2009: 68-70, De caelo 290b10-291a25). Bewegte Körper können andere Körper in Bewegung versetzen und schließlich auch die Seele bewegen. Bis zum Ende der Renaissance sprach die Ästhetik vorwiegend von dieser körperlichen Wirkung der Musik. Mit dem Ideal des »imitare le parole« Gioseffo Zarlinos (1562: 340, Buch 4, Kap. 33) und der Auffassung, Musik verfüge über einen Zeichenvorrat, der dem der Rhetorik ähnlich sei, setzte sich im 16. und 17. Jahrhundert langsam die Auffassung durch, Musik sei nicht nur der Sprache ähnlich, wie man es schon im Mittelalter gelegentlich findet, sondern eine eigene Sprache (zuerst bei Mersenne 1636: Proposition 47), sie habe also nicht nur Wirkung, sondern auch Bedeutung - und zwar auch ohne Unterstützung durch den Text. Mit den Komponisten der Vorklassik verbreitet sich in der ersten Hälfte des 18. Jahrhunderts schließlich die Vorstellung, dass der Komponist nicht mehr objektive, an konkreten Zeichen festzumachende Affekte, sondern seine individuellen, subjektiven Empfindungen in der Musik darzustellen, zur Sprache zu bringen habe. Damit wird die Komposition endgültig zum Ausdruck der intellektuellen Individualität des Komponisten. 
Auch in der Operngeschichte gibt es das Oszillieren zwischen den Polen Ratio und Körper. In der Opera seria des Barock sind Komposition, Libretto und Bühnendarstellung stark formalisiert und rational durchstrukturiert. Das Publikum des 18. Jahrhunderts wird jedoch durch die Kunst der Sänger zur Raserei getrieben. Es ist bezeichnend, dass die größte körperliche und erotische Ausstrahlung ausgerechnet von Kastraten ausging und bis heute ausgeht (vgl. Ortkemper 1993). Ihre körperliche Abnormität lenkte und lenkt die Aufmerksamkeit der Betrachter unmittelbar auf den Körper, genauso wie ihre ungeheuren stimmlichen Fähigkeiten. Die Anmaßung der Kastraten, das Äußerste öffentlich aus ihren Körpern herausholen zu dürfen, sich in ihrer ganzen extremen Körperlichkeit und Emotionalität öffentlich zur Schau zu stellen und in ihrer körperlichen Wirkung die intellektuellen Leistungen des Komponisten weit in den Schatten zu stellen, war - so kann man spekulieren - nur um den Preis einer geschlechtlichen Neutralität möglich. ${ }^{8}$

Im Verlauf des 19. Jahrhunderts schwang sich die Instrumentalmusik zur beherrschenden Gattung über die Vokalmusik auf. Die Autonomieästhetik eines Eduard Hanslick machte die Musik als tönend bewegte Formen unabhängig von jeglicher körperlicher Individualität. Gleichzeitig ist das 19. Jahrhundert jedoch auch die Blütezeit der Instrumentalvirtuosen, die die Aufmerksamkeit des Publikums von der Form des musikalischen Werkes ablenkten und auf ihre individuellen körperlichen Fähigkeiten und - die vielen Karikaturen belegen es - auf ihr Aussehen richteten. Öffentlich dargestellte individualisierte Körperlichkeit ist bis heute eine Provokation für die Gesellschaft; brillanten Denkern und Autoren, Komponisten komplexer mehrstimmiger Musik wird nicht selten die göttliche Inspiration zugeschrieben. Mit der Genieästhetik des ausgehenden 18. Jahrhunderts werden sie endgültig zu Schöpfern origineller, weil individueller Werke. Da sie ihre Schöpfungen schriftlich niederlegen, erscheinen diese entkörperlicht, rein geistig. Men-

8 Die Stars der Oper des 18. Jahrhunderts und ihre Beziehung zum Publikum haben viele Parallelen mit dem Kommunikationssystem der populären Musik der Gegenwart. Eine ähnlich zurückgenommene Geschlechtlichkeit weisen zahlreiche männliche Popstars besonders der letzten 40 Jahre auf. Androgyn geschminkt und gekleidet, mit hohen Stimmen und ohne eindeutig betonte Geschlechtsmerkmale treiben Sänger von Boy George bis Bill Kaulitz die Individualisierung der Geschlechtlichkeit auf eine Spitze. Sie entschärfen - vor allem im Fall von Teeny-Stars - mit dieser deutlich erkennbar gemachten Fiktionalisierung jedoch auch die Zumutungen für die Kommunikation, sodass sie auch und gerade für Rezipienten attraktiv werden, für die allzu deutlich präsentierte Geschlechtlichkeit problematisch ist. Andererseits können Zweifel über eine Geschlechtszugehörigkeit die Aufmerksamkeit für die Individualität eines dargestellten Körpers noch erhöhen, wie in den Fällen z.B. der Images von Amanda Lear oder Lady Gaga. 
schen dagegen mit herausragenden körperlichen Begabungen, Virtuosen der Stimme, eines Instruments oder des Tanzes, die mit ihrer Kunst auf den öffentlichen Auftritt angewiesen sind, wurden stets auch mit Argwohn betrachtet. Es gibt keine Teufelskomponisten, wohl aber Teufelsgeiger. 9

Mit der Erfindung der Schall- und Bildaufzeichnung konnten erstmals auch die Handlungen des Musikmachens in ihrer ganzen Körperlichkeit wiederholbar gemacht und dadurch zeitlich fixiert werden. Die Notenschrift hatte ihr Monopol auf die Produktion zeitlos gültiger Werke verloren. Hiermit änderte sich nach und nach auch die Rezeption von Musik. Analysiert man die musikalische Kommunikation der Gegenwart, kann man zwei unterschiedliche Arten feststellen, über und mit Musik zu kommunizieren: Eine, in der die Individualität und Einzigartigkeit der Komposition im Vordergrund steht, und eine, die um die Individualität des Musikers kreist. In der ersten geht es um Kunst, um die Frage, wie ein Komponist sein Medium, sein Material, in eine bestimmte, originelle und individuelle Form gegossen hat, sodass es dem Rezipienten Fragen aufgibt, die er durch Verstehensprozesse zu beantworten sucht. Diese Musik wird unabhängig von jeglicher Körperlichkeit gedacht. Sie materialisiert sich in den geschriebenen Noten, muss aber auch zum Klingen gebracht werden. Da es sich bei diesen Realisierungen der Noten jedoch nur um Interpretationen des Willens des Komponisten handelt und nicht um das Stück selbst, steht das eigentliche »Werk « über der Körperlichkeit der ausführenden Interpreten.

In der zweiten Art, über und mit Musik zu kommunizieren, geht es um das, was oft als »das Populäre Noten spielen in diesem kommunikativen System keine wahrnehmbare Rolle. Hier kommunizieren Musiker mit Hörern, hier kreist alles um deren Individualität. Der populäre Hörer - ich verwende diesen Begriff, um deutlich zu machen, dass man alle Arten von Musik ähnlich wahrnehmen kann kümmert sich nicht um die Art und Weise, mit der ein Schöpfer sein Medium formte - er hört in den Tönen keine Fragezeichen. Er nimmt vor allem die individuelle Körperlichkeit wahr: die einzigartige Stimme der Sängerin, ihre eigene Art zu singen, den Klang der Stradivari oder Stratocaster, also das,

9 Dieser Unterschied galt allerdings nicht für Frauen. Sängerinnen und Musikerinnen standen grundsätzlich unter moralischem Verdacht. Aber auch Komponistinnen, die ihre Werke veröffentlichten, provozierten das Anstandsempfinden. Seit dem 16. Jahrhundert, mit einem Höhepunkt im 19. Jahrhundert, hatte sich die Ansicht durchgesetzt, dass ehrbare Frauen auf keinen Fall öffentlich sichtbar werden durften, wenn sie nicht zu öffentlichen Frauen erklärt werden wollten. Die amerikanische Komponistin Amy Beach z.B. gab nach der Hochzeit weitgehend die Veröffentlichung ihrer Kompositionen auf, Alma Mahler sogar alle Kompositionsversuche. 
was man als Sound bezeichnen könnte, aber auch die Äußerlichkeit der körperlichen Erscheinung des Musikers und seiner Instrumente. Und die Musiker achten darauf, dass sie diese Elemente besonders hervorheben, um sich von anderen Musikern abzugrenzen. Populäre Musik ist nicht deshalb körperlich, weil sie einen regelmäßigen, deutlich wahrnehmbaren Rhythmus hat und man danach tanzen kann - eine mitreißende Motorik haben auch Stücke der so genannten »klassischen Musik«. Doch während der Kunstdiskurs versucht, idealerweise alle Kommunikation auf das Problem der Differenz von Medium und Form in der Komposition zu lenken, indem er selbst in der Konzertsituation Körperlichkeit so weit wie möglich in den Hintergrund der Wahrnehmung drängt und immer wieder durch komponierte Irritationen die Aufmerksamkeit des Hörers auf die Machart des Stückes lenkt, richtet das Kommunikationssystem der populären Musik alle Aufmerksamkeit auf den Körper und die individuellen Handlungen, die die klingende Musik hervor bringen. Diese grundsätzliche Spaltung der Diskurse wurde durch Medien möglich, die - anders als die Schrift als Medium des Geistes - die Abbildung von Körperlichkeit ermöglichten: die Schall- und Bildaufzeichnung. Fanmagazine, Fernsehauftritte und Videoclips sind nur die (vorerst) letzte Konsequenz aus dem Bedürfnis nach Körperlichkeit des Diskurses, das durch Schallaufzeichnungen nur zum Teil gestillt werden kann (s. ausführlicher zu den beiden Kommunikationssystemen Helms 2008).

Die Medien der Schall- und Bildaufzeichnung haben deutliche Auswirkungen auf die mit dem Machen von Musik verbundene Körperlichkeit. Mit der Schallplatte und dem Videoclip entwickelte sich auch das Musikmachen - historisch lange nach dem Komponieren - von einer Dienstleistung für ein mehr oder weniger bekanntes Publikum, dem man in Faceto-Face-Kommunikation gegenüber stand, zu einem Produkt, einer Ware für ein Publikum, über dessen Zusammensetzung man bei der Produktion nur spekulieren kann, einer Ware zudem, die in der Kommunikation ein Feedback des Publikums zu großen Teilen nur über das Medium Geld erlaubt. Als die Musik in Form von gedruckten Noten ab dem 16. Jahrhundert sich einen Markt schuf, hatte das die Entwicklung des Kunstsystems beschleunigt. Auf dem Markt setzten sich auf Dauer große Namen durch, die ihre Einzigartigkeit durch die Individualität ihres notierten Werkes, ihres geistigen Outputs, belegt hatten. Aufgabe des Publikums war es, diese Individualität im Werk wahrzunehmen - was unweigerlich und bereits im 19. Jahrhundert zu Verstehensproblemen führte. Auf dem Markt der Träger von Schall- und Bildaufzeichnungen dagegen setzt sich die Einzigartigkeit körperlicher Eigenschaften der Musiker durch: die Individualität der Stimme einer Björk oder eines Luciano Pavarotti, das Aussehen einer 
Anna Netrebko oder eines Mick Jagger, die Fingerfertigkeit eines David Garrett oder eines Stevie Ray Vaughan. Je größer das Angebot auf dem Markt ist, desto schwieriger fällt den einzelnen die Abgrenzung von ähnlichen Produkten. Da CD, Bild und Film Körperlichkeit abbilden, können sie sich nur dann von anderen Produkten unterscheiden, wenn diese Körperlichkeit weiter individualisiert wird - bis zur Exzentrizität von Stimme, Spiel, Aussehen und Verhalten. Die Beschleunigung einer körperlichen Individualisierung führte nicht zu Problemen mit dem Verstehen des musikalischen »Materials « im traditionellen Sinne. ${ }^{10}$ Statt an die Grenzen von Bildung und bewusstem Hören geht diese Musik an die Grenzen, die unsere Gesellschaft errichtet hat, um das öffentliche und unmittelbare Ausleben körperlicher Individualität zu verhindern, an die Grenzen von Geschmack, Moral und Gesetzen, aber auch an die Grenzen des körperlich noch Möglichen. Sie kündigt die Subtilität einer Erotik auf, die eigentlich durch Riten der Liebeswerbung, durch Moral und Gesetze dafür sorgt, dass das Ausleben des Sexualtriebs (teilweise bis zur Unwahrscheinlichkeit) verzögert und aus der Öffentlichkeit verbannt wird. Sie kündigt auch die Subtilität einer Machtdarstellung auf, die durch ähnliche Regulative das Ausleben körperlicher Gewalt unwahrscheinlicher macht.

Ihre Provokationen gesellschaftlicher Grenzen sind nur deshalb ohne allzu großes Gefährdungspotential, weil sie im Kontext einer doppelten Fiktionalisierung geschehen. Zunächst macht die Musik deutlich, dass es sich um eine Welt außerhalb des Alltags handelt. Hinzu kommt bei den Bild- und Schallmedien die Tatsache, dass die hierauf hör- und sichtbar gemachten Handlungen im Gegensatz zu realen Handlungen identisch wiederholbar sind und dass die Musiker trotz aller Nähe ihrer Abbildungen unfassbar fern sind. Vor der Erfindung der Schall- und Bildaufzeichnung wurde die Provokation, die das Vorführen von individueller Körperlichkeit für die Gesellschaft bedeutete, durch das ganz offensichtliche Agieren der Musiker in einer fiktiven Rolle gemildert, in der Oper und im Kunstlied z.B., in denen die Sänger auch noch als Interpreten eines Komponisten geschützt waren, genauso wie in der englischen Music Hall des 19. Jahrhunderts, in der die Sänger zwar bereits statt einer vom Komponisten und Texter geprägten Rolle ein individuelles Image entwickelten, das jedoch auf groben Klischees wie dem »Coon«, dem Iren, dem Cockney, dem Dandy usw. basierte, sodass stets wahrnehmbar blieb, dass sich der Sänger in eine Rolle begibt. Die modernen Medien machen diese Rollenkennzeichnung überflüssig; der Musiker der Gegenwart tritt mit einem »Image«, einer Fiktionalisierung seiner selbst

10 Dementsprechend braucht es auch eigentlich keine Musikwissenschaft, um Musik im System des Populären für ihr Publikum zu erklären. 
auf. Die Individualität, die mit Hilfe der Medien der Schall- und Bildaufzeichnung entwickelt wird, täuscht eine Übereinstimmung mit dem realen Ich der Sängerin oder des Sängers vor. Das Image entsteht durch die Notwendigkeit der Individuation, die, um erfolgreich zu sein, d.h. um sich zu unterscheiden, deutlich die Grenzen der Alltagskommunikation überschreiten muss - z.B. auch durch überdeutliche Zeichen der Erregtheit oder der Gewaltbereitschaft - und den Spielcharakter bzw. den fiktiven Charakter der Kommunikation deutlich macht. Gleichzeitig wird diese Kommunikation jedoch immer wieder an ihrem Realitätsbezug - d.h. am tatsächlichen Ich der Sängerin oder des Sängers - gemessen. In der Welt der populären Musik gelten nicht die Grenzen von Moral und Gesetz. Eine Regulation findet lediglich durch die Diskussion um die Glaubwürdigkeit bzw. Authentizität des Images statt (vgl. Helms 2008: 89-91). Während eine Opernsängerin die Rollen der verführerischen Salome oder der Lulu mit dem Kostüm in der Garderobe ablegen kann, ist die Sängerin im System der populären Musik gezwungen, in der Öffentlichkeit auch außerhalb der Musik die Fiktion ihres Images weiter zu spielen, um authentisch zu wirken. Diese Annäherung an den Alltag ermöglicht es erst, dass sich das Publikum mit den Sängern identifiziert, sie zum Mittel der eigenen Individuation wählt. Das muss nicht unbedingt zur Auflösung von Moral und Anstand führen, solange unterschieden wird zwischen Kommunikation des Alltags und Handlungen der Stars, die in einer kommunikativen Sonderwelt existieren, unerreichbar abgeschirmt durch die Massenmedien.

So braucht es nach wie vor Sonderwelten, markiert durch Musik, in denen sich Fans über die strengen Regeln der Alltagskommunikation herausheben und ihren Stars nahe sein können. Auf der Party z.B. ist körperbetonende Kleidung ein geringeres Problem und körperliche Anmache beim Tanz erlaubt. Abgeschottet durch den Kopfhörer kann man sich mit dem angebeteten Star für die Dauer des Songs vereint fühlen. Die Medien der Schallaufzeichnung haben es allerdings, das zeigt das letzte Beispiel, möglich gemacht, dass diese Sonderwelten des Festes immer alltäglicher, dass die Grenzen zwischen Fest und Alltag fließend werden. Portable Abspielgeräte entrücken den Gang durch die Stadt aus dem Alltag, Musik im Modeladen will das Einkaufen zur Party machen, Friseure und selbst Zahnärzte entspannen ihre Arbeit am fremden Körper durch Musik. Gleichzeitig drängt auch die massenmediatisierte Musik in die privatesten Bereiche der Kommunikation. Mit der Hilfe von Musik und der von ihr geschaffenen Sonderwelt mögen Grenzen körperlicher Kommunikation leichter überschritten werden, weil der Alltag ausgeblendet wird. Musik hilft durch ihre eigene Nahwelt, die Nahwelt zwischen Paaren aufzubauen und die Umwelt auszugrenzen. Sie 
hilft - vielleicht auch durch Identifikation mit dem Star, dem oder der grenzüberschreitendes Verhalten erlaubt ist - dem Partner eine Körperlichkeit vorzuzeigen und vorzuspielen, die man sich im Alltag nicht (zu-)getraut hätte. Und sie schafft im Nachhinein Symbole der Gemeinsamkeit, des Weißt-du-noch, wann immer das Lied später noch einmal erklingt.

\section{Was ist erotische Musik?}

Die Frage nach dem, was erotische Musik sei, beantworten unterschiedliche Kulturen sehr verschieden. Wir haben oben gesehen, dass Kleriker des Mittelalters jede Darstellung von Individualität in der Musik, sei sie körperlicher oder intellektueller Art, als Sitten verderbend und lüstern angesehen haben. Für Anhänger mancher religiöser Konfessionen ist auch heute noch jede Art von vorgeführter Musik, die nicht der Rezitation geistlicher Texte dient, ein Skandal verbotener Sinnlichkeit - hier sei nur an die Verfolgung von Musikern, Musikhändlern und Musikhörern im Afghanistan der Taliban erinnert.

Ich möchte zur Klärung der Frage nach der erotischen Musik drei Kategorien musikalischer Zeichensysteme und zwei alltagssprachliche Begriffsverwendungen unterscheiden. Rein musikalische Zeichen der Erotik verwenden Kompositionen, die Erotik symbolisch in den Noten darstellen, Kompositionen, die Erotik ikonographisch repräsentieren und schließlich die Repräsentationen körperlicher Identität durch Musiker als Teil ihres Sounds. Alltagssprachlich wird auch Musik mit erotischen Texten und Musik, die aus unterschiedlichen Beweggründen vor dem oder beim Geschlechtsverkehr genutzt wird, als erotische Musik bezeichnet. Als erotische Musik im strengen Sinne kann allerdings nur die dritte Kategorie verstanden werden.

Für den Musikwissenschaftler naheliegend sind zunächst Kompositionen, deren Noten Symbole für Erotik enthalten. Das Liebesduett aus dem zweiten Akt von Richard Wagners Tristan und Isolde ist sicherlich das bekannteste Beispiel einer symbolischen Darstellung eines erotischen Moments. Die Gesangslinien von Isolde und Tristan, zu Beginn bei "Sink hernieder, Nacht der Liebe« (2. Aufz., 2. Szene) noch durchaus individuell gestaltet, teilweise auch bewusst gegenläufig und kontrastierend, gleichen sich, wie ein zögerndes Zieren, immer weiter an, versuchen - freilich vergeblich - die Verschmelzung in Textgleichheit und Unisono zu erreichen (Wagner 1992: 145158, s. z.B. Takte 1122-1161). Im Orchester erklingt das "Sehnsuchtsmotiv«, aber warnend auch immer das »Tagmotiv«. Um die komponierte Symbolik 
dieser Szene, den Versuch gegenseitiger Annäherung zweier Individuen zu erkennen, braucht es die musikalische Analyse, den Einsatz des Intellekts.

Dass sich die Bedeutung der Szene aus Tristan und Isolde auch dem musikalisch weniger Gebildeten erschließt, dafür sorgen nicht nur die Inszenierung, nicht nur der Text (mit »nimm mich auf in deinen Schoß ist wohl nicht nur die besungene »Nacht der Liebe« gemeint), sondern auch die Gestik bzw. Motorik der Musik. Am Ende des zweiten Auftritts, an dem sich Isolde und Tristan dem Libretto nach so weit nähern, dass sie meinen, mit ihrer Identität in der Identität des jeweils anderen aufzugehen (»Tristan du, ich Isolde, nicht mehr Tristan«; Wagner 1992: 209-219, Takte 1575-1630), schraubt sich die Komposition in immer engeren Kreisen und immer kürzeren Phrasen in immer größere Höhen und immer größere Lautstärke. Alles drängt kompositorisch auf eine Entspannung, eine Auflösung hin - die dann doch nicht kommt. Hier handelt es sich nicht mehr nur um symbolische, sondern auch und vorrangig um ikonograpische Zeichen, d.h. um eine tonmalerische Darstellung. Die Bewegung der Musik repräsentiert hier direkt und unmittelbar nachvollziehbar die Bewegung einer sexuellen Ekstase.

Nicht erst seit Blake Edwards' Film Zehn - Die Traumfrau (USA 1979) gilt Maurice Ravels Ballet pour orchestre Boléro (UA 1928) als die Darstellung des »élement musico-sexuel émanant de l'obsession musicale«, wie der Komponist selbst sich ausdrückte (Jourdan-Morhange 1945: 166). Freilich basiert fast jede Art von komponierter Musik auf dem Prinzip von Spannung und Entspannung: Dissonanz und Auflösung, Beschleunigung und Verlangsamung, Crescendo und Decrescendo, Verdichtung und Leere. Ravel komponierte mit dem Boléro ein gut 15-minütiges Crescendo mit ostinatem Rhythmus und abruptem Schluss auf dem Höhepunkt. Erotisch wird das Stück erst, wenn der Zuhörer interpretierend die Parallelen zwischen den Bewegungen der Musik und denen des Geschlechtsverkehrs zieht. Erst der Kontext liefert sexuelle Konnotationen: ein Liedtext, die Handlung einer Oper oder eines Films, die Bewegungen einer Interpretin oder eines Interpreten, der Titel. Wer wüsste sonst, um was es gehen soll beim Hören oder Spielen von Edvard Griegs Lyrischem Stückchen op. 43, Nr. 5, mit dem Titel »Erotik«? Ein Schelm also, wer Arges denkt beim Weben tönend bewegter Formen.

In Noten symbolisierte Körperlichkeit und Annäherung sowie die auskomponierte ikonographische Nachzeichnung von Bewegungen sind nicht an sich erotisch, sondern vermitteln Erotik auf dem Umweg des Verstehens, der Interpretation, also eines intellektuellen Prozesses. Es handelt sich hierbei um Erotik in der Komposition, nicht aber um erotische Musik. Tatsächlich erotische Musik, meine dritte Kategorie, wird nicht komponiert, sondern gemacht. In erotischer Musik wird die geschlechtliche Körperlichkeit der 
Musiker selbst repräsentiert, durch sie führt der Musiker seine geschlechtliche körperliche Identität dem Hörer vor. Will man in der hier gewählten Zeichensystematik Charles S. Peirces bleiben (2000: 147-159; vgl. Karbusicky 1986: 13f.), kann man von indexikalischen Zeichen sprechen. Sie verweisen unmittelbar und direkt auf den Körper des Musikers und setzen keine musikalische Bildung, nur mal mehr, mal weniger Lebenserfahrung voraus. Dem Komponisten ist diese Form der Erotik nicht zugänglich. Er ist hierzu auf seine Interpreten angewiesen, auf Musiker und auf deren Bereitschaft, ihre eigene Körperlichkeit einzubringen. Im Kommunikationssystem des Populären ist die Darstellung der Körperlichkeit der Musiker zentrales Ausdrucksmittel, das den Sound produziert, die Differenz des Musikers zu anderen Musikern. Repräsentativ für den ganzen Körper steht hier die Stimme, die dem Hörer nicht nur das Geschlecht verrät, sondern auch - auf der Basis seiner eigenen Erfahrungen - Anhaltspunkte für Körpergröße, Alter, Ethnie, soziale Zugehörigkeit und emotionale Gestimmtheit liefern kann (Frith 1998: 183-202). Auch das räumliche Verhältnis der Körper von Musiker und Hörer zueinander wird angedeutet: Eine laute oder gar schreiende Stimme stellt Distanz her; eine leise Stimme produziert dagegen Nähe, die noch gesteigert werden kann, wenn sie sehr präsent in den Vordergrund des Stereoklangraums gemischt und nur wenig oder gar nicht verhallt wird. Eine individuelle Körperlichkeit erhält die nahe Stimme vor allem durch ihren Anteil an Geräuschen: Atemgeräusche vor allem, hörbare Lippenbewegungen, Dinge, die man ansonsten nur wahrnimmt, wenn das Gegenüber wirklich nah, auf Flüsterdistanz ist (vgl. Kreutz 1997: 305). Dazu kommen stimmliche Zeichen, die - nicht zuletzt durch die Medien als Signale verbreitet und kulturell geprägt - sich als Symbole der Erotik eingebürgert haben: ein Hauchen oder kehliges Knurren z.B. bis hin zum Stöhnen, die gespielte Unterordnung in der künstlichen Kinderstimme einer erwachsenen Frau oder der beschwichtigende Klang einer relativ hohen, leisen Männerstimme... Stilisiert finden sich ähnliche Signale der vokalen Erotik auch im Instrumentalspiel, besonders deutlich in Instrumenten, die der menschlichen Stimme nahe kommen, die hauchen können, knurren, stöhnen oder schreien. Instrumentale Virtuosität und vokale Stärke repräsentieren aber auch körperliche Überlegenheit und Stärke des Musikers. Gerade im Bereich männlicher Erotik - wohlgemerkt nicht explizit männlicher Musiker, denn auch Frauen können sich dieser Symbolik bedienen - sind die Grenzen der Darstellung körperlicher, geschlechtlicher Individualität fließend: Erotik kann durchaus auch in die Repräsentanz von körperlicher Macht (mit der dahinter stehenden Androhung von Gewalt) umschlagen und so können 
Stimmen, die nach körperlicher Stärke klingen, durchaus auch als Zeichen für Gewaltbereitschaft verstanden werden.

Musikstücke mit Texten, die von Geschlechtsverkehr handeln, weisen nicht unbedingt auch Erotik in der Musik auf. Viele Stücke in der Tradition der liederlichen Lieder sind Kontrafakte eines nicht-erotischen Repertoires, ein typisches Beispiel hierfür sind die Repertoires der Ohne Hemd und ohne Höschen-Reihe der Polydor, die bekannten Party-Melodien eindeutige Texte unterlegte. Auch die Musik eines Mickie Krause ist alles andere als erotisch, sondern funktioniert als Partymusik vermutlich nach dem bereits beschriebenen Phänomen der Produktion von Gemeinsamkeit durch gemeinsamen Tabubruch. Geschieht dieser Tabubruch heimlich, durch die Verwendung von sprachlichen Bildern, die nur Eingeweihten bekannt sind, ist dieser integrierende Effekt besonders stark (vgl. z.B. die Texte im Blues im Beitrag von Thomas Phleps in diesem Band). In Songtexten kann durchaus auch eine individuelle geschlechtliche Körperlichkeit präsentiert werden, allerdings immer als indirekter Verweis und nicht in der körperlichen Unmittelbarkeit, zu der erotische Musik fähig ist.

Als letztes gilt es, die Musik zu diskutieren, die für die Begleitung von Geschlechtsverkehr genutzt wird. Auch wenn es hierzu keine gesicherten Forschungen gibt (vgl. den Beitrag von Michael Ahlers und Christoph Jacke in diesem Band), möchte ich aus kommunikationstheoretischer Perspektive behaupten, dass diese tunlichst weder allzu deutlich Erotik in der Komposition noch im Musikmachen enthalten sollte, sondern eher unerotisch im hier verwendeten engen Sinn daherkommen muss. Solange es noch um die Anbahnung eines Einverständnisses geht, kann der Spielcharakter, den Musik schafft, kann das Vorspielen von Erotik durch eine/n Dritte/n durchaus hilfreich sein. Musik kann Stimmung machen, anfänglich auch bei der Verhaltenssynchronisation helfen. Spielerisch haucht man zusammen mit Jane Birkin »Je t'aime« und kann doch noch jeder Zeit zurück - sollte der Partner z.B. mit »moi non plus « antworten. Doch irgendwann muss der Schritt aus der Fiktion der musikalischen Sonderwelt heraus gewagt werden, und dann kann die Nahwelt zwischen den Partnern durch einen Dritten, der mit seiner körperlichen Individualität allzu deutlich, wenn auch nur aus den Lautsprechern der Stereoanlage auf sich aufmerksam macht, gestört werden. Ähnliches gilt für die Kategorien von Erotik in der Komposition. Kompositorische Strukturen, die die Aufmerksamkeit der Hörer auf sich ziehen, die durch Irritation Analyse und Interpretation einfordern, lenken ab und zerstören die Nahwelt. Der Boléro stört, wenn die Bewegungen, die die Musik repräsentiert, den Bewegungen der Hörer zuwider laufen. Sex ist kein Ballett mit einer Choreographie, die ein Dritter festlegt. Die Vereinigung 
von Körper und Geist mit Richards Hilfe, wie Clothwig Poth (1982) seinen Roman einer Jagd nach dem ultimativen Sex mithilfe des Liebesduetts aus Wagners Tristan nannte, muss auch an der musikalischen Struktur scheitern, die sich in die kommunikative Nahwelt der Partner drängt.

Musik stimuliert Ekstasen durch die Herstellung völliger Gleichsinnigkeit, durch das Aufgehen des Ichs in sich selbst in einer Gemeinschaft (s.o.). Während beim Tanz diese Gleichsinnigkeit zwischen Musikern und Tänzern durch den Rhythmus hergestellt wird, braucht die Entstehung der erotischen Nahwelt eines Paars viel mehr als rhythmische Bewegungen, um Gleichsinnigkeit deutlich werden zu lassen. Der Musiker als virtueller Dritter im Schlafzimmer kann nichts zur Entstehung dieser Nahwelt aus gegenseitiger Darstellung und Akzeptanz körperlicher Lust beisteuern. Wird er wahrgenommen, droht er sogar die Nahwelt zu zerstören. So spielt Musik schließlich nur noch die Rolle einer Klangtapete, die störende Umweltgeräusche fernhält und verhindert, dass der Zauber von außen zerstört wird. Sie darf daher nicht zu deutlich Individualität repräsentieren, weder kompositorisch-intellektuell noch musikalisch-körperlich. Sie muss eben »Kuschelrock« bleiben.

\section{Wozu braucht (populäre) Musik Erotik?}

Kommunikationssysteme, die musikalische Medien wie Töne oder Sound (im Sinne von Helms 2003) verwenden, müssen zum Teil sehr komplexe Strukturen annehmen, damit die Schließung des Kreislaufs der Kommunikation, des Austauschs reziproker Information bzw. Handlungsorientierung gelingt. Anders als bei der Kommunikation zwischen Komponisten und ihren Interpreten, die auf der Basis desselben Mediums, des Tons, kommunizieren können, braucht die Kommunikation zwischen Musikern und Hörern unterschiedliche Medien, um zustande zu kommen. Der Unterschied zwischen Musikern und Hörern ist ja gerade dadurch definiert, dass erstere in Tönen und Sound kommunizieren, letztere aber nicht entsprechend antworten dürfen. Sie können sprachlich analytisch auf Töne und Sound reagieren, erwarten kann der Musiker eine solche Reaktion, die eine gewisse Bildung beim Gegenüber voraussetzt, jedoch nicht - oder doch nur von spezialisierten Mitgliedern des Publikums: den Konzertkritikern. In Konzerten sind Musiker auf die Beobachtung der Körpersprache ihres Publikums angewiesen, um festzustellen, ob ihre Mitteilung ankommt. Dabei können sie nur feststellen, dass es ihnen gelungen ist, eine Nahwelt um sich und das Publikum herum zu produzieren. Was sie beobachten können, ist lediglich die Aufmerksam- 
keit ihrer Hörer, den Grad, in dem diese auf die Bühne und auf die Handlungen, die dort stattfinden, fixiert sind. Gelingt es ihnen, das Publikum ganz in ihren Bann zu ziehen, entstehen auf beiden Seiten Flow-Erlebnisse, das Gefühl, ganz in der Musik, in der Gemeinschaft einer Nahwelt, aufzugehen. In der Kunstmusik wird die Kommunikation zwischen Musikern und Hörern durch einen hohen Grad an Entkörperlichung schwer gemacht: formale Kleidung, ein Publikum, das sich nur minimal bewegen und keine Lautäußerungen von sich geben sollte, Musiker, die sich zwar bewegen dürfen, um sich gegenseitig abzustimmen, aber nicht, um das Publikum zu Bewegungen zu animieren. Die Steifheit des bürgerlichen Konzerts ist gewollt, weil hier in der Kommunikation noch ein Dritter mitgedacht wird, der mit großer Autorität und Distanz Entscheidendes mitzusprechen hat: der Komponist. Die Musiker als Verkünder seines Willens stehen nicht für sich selbst, sondern interpretieren sein Werk. Der intellektuelle Prozess, der dieser Kommunikation als Ideal zugrunde liegt, drückt sich in der Unterdrückung von Körperlichkeit aus, die lediglich als symbolische und ikonische Zeichen verschlüsselt in der Komposition deutlich werden darf.

Die Kommunikation in einem Konzert im System des Populären dagegen ist wesentlich direkter: Hier stehen die Musiker für sich selbst und müssen dementsprechend auch als Individuen wahrgenommen werden. ${ }^{11}$ Das Medium Sound, das dazu dient, Musiker voneinander zu unterscheiden (Helms 2003), verweist vor allem auf Körperlichkeit: der individuelle Klang einer Stimme (oder eines Instruments), die Abmischung und Effekte wie Hall und Echo, die um die Körper von Musikern und Hörern einen virtuellen Raum errichten. Angesichts wachsender Konkurrenz der Musiker, die durch die Schallaufzeichnung noch extrem verstärkt wurde, weil hierdurch jeder immer und gleichzeitig mit allen anderen konkurrieren muss, wird Individualisierung zu einem drängenden Problem der Kommunikation. Diese Individualisierung funktioniert für Musiker nur durch das überdeutliche Vorzeigen des Körpers, eine individualisierte Stimme und ein individualisiertes Aussehen. Die Präsentation der individuellen Geschlechtlichkeit, d.h. Erotik, provoziert nach außen, stößt $a b$, nach innen verstärkt sie jedoch die Bindung. ${ }^{12}$ Dadurch, dass das Gegenüber die Enthüllung des Körpers toleriert, sich nicht abwendet, entsteht ein Einverständnis, das auf seine virtuelle Art fast intim zu nennen ist. Solche scharf gezogenen Grenzen sind vor allem deshalb wichtig, weil im Kommunikationssystem der populären Musik nicht

11 Oder - wie im Falle von Coverbands - als Klons von Individuen, nicht aber als Interpreten.

12 Dasselbe gilt auch für die körperliche Darstellung von Macht, auf die der Beitrag hier nicht eingeht. 
nur die Individuation der Musiker, sondern auch die der Hörer wichtig ist. Sie können sich mit provozierender Musik von Fans anderer Bands oder von anderen Generationen absetzen.

Anders als in der Alltagskommunikation, in der heterosexuelle Kommunikanden in der Regel nur gegengeschlechtliche Enthüllungen des lustvollen Körpers tolerieren, scheint in der Situation des Konzerts, in der wenige (Musiker) mit vielen (Zuhörern) kommunizieren, auch Gleichgeschlechtlichkeit zu funktionieren. Auch die Beobachtung eines intimen Verhaltens, dass man nicht auf sich selbst beziehen will, scheint Nahwelten des Einverständnisses produzieren zu können. Die intime Kenntnis eines Kommunikationspartners scheint bis hin zum imaginierten Rollentausch, zur Identifikation gehen zu können. ${ }^{13}$ Für die Dauer des Songs kann man sich den imaginierten Körper des Musikers als Gegenüber vorstellen, sich jedoch auch in ihn hineinprojizieren, sodass man ggf. dessen Erotik auf den eigenen Körper überträgt. Musikalische Kommunikation besteht nicht aus dem sonst üblichen Hin und Her, aus Frage und Antwort, Position und Gegenposition der Alltagskommunikation, durch das sich Kommunikationspartner wahrnehmbar voneinander unterscheiden. Musik erfordert die gegenseitige Beobachtung von Gleichsinnigkeit: geistiges oder auch körperliches Mittun. Die Identifikation mit den Musikern bzw. die Kommunikation der Gleichsinnigkeit nach außen durch Imitation des Verhaltens der Musiker (z.B. Luftgitarre spielen), ist daher ein zu erwartendes kommunikatives Verhalten. Ähnliches gilt für Erotik. Auch die Präsentation von Körperlichkeit in der Kommunikation erfordert, wenn sie akzeptiert wird, eine gleichsinnige Antwort. Erotik ist mit Aufmerksamkeit, dann aber auch mit eigener Erotik zu beantworten - das gilt auch für die fiktionalisierte Erotik musikalischer Kommunikationssysteme, die mit gespielter oder auch nur imaginierter Erotik beantwortet werden kann. Musik und Erotik sind ansteckend.

Bisher habe ich von Konzertsituationen gesprochen und damit auch virtuelle Konzertsituationen wie das Abspielen einer Schallaufzeichnung

13 Dieses Phänomen ist vor allem wichtig, wenn nicht Erotik, sondern Macht durch die Darstellung körperlicher Gewalt mitkommuniziert wird. Gestik und Mimik im Rap z.B. symbolisieren kaum noch unterschwellig körperliche Überlegenheit und Gewaltbereitschaft. Diese Provokation bewirkte in einer Alltagskommunikation entweder eine Unterwerfungsreaktion oder Flucht bzw. Gegengewalt. Keine dieser Reaktionen kann im Kommunikationssystem der populären Musik gewollt sein. Die Tatsache, dass HipHop als Kommunikation dennoch funktioniert, könnte darauf zurückzuführen sein, dass sich Hörer besonders intensiv mit den Musikern identifizieren, die Richtung der Kommunikation ändern, indem sie sich statt als Angesprochene als Sprecher sehen und ihre Umwelt zu den Angesprochenen machen, gegen die sich die Androhung von Gewalt richtet. 
gemeint. Einige Probleme werden durch die musikalischen Massenmedien allerdings gegenüber dem Konzert verschärft. Die Face-to-Face-Kommunikationssituation im Konzert macht eine unmittelbare Interaktion zwischen den Kommunikanden möglich. Die Musiker können in gewissen Grenzen unmittelbar auf die Reaktionen ihres Publikums reagieren. Diese Unmittelbarkeit ist im Fall der Kommunikation über die Massenmedien der Schallaufzeichnung nicht möglich. Musiker erfahren nichts von den Reaktionen ihres Publikums beim Hören der Songs einer CD. Das Zustandekommen von Kommunikation, der Erfolg der musikalischen Mitteilung der Musiker, schlägt sich für sie in Zahlen nieder: Verkaufszahlen, Charts, Airplay, Einschaltquoten. Diese Statistiken messen allerdings lediglich die Aufmerksamkeit der Hörer, nicht jedoch, wie sie reagiert haben, oder gar, was sie sich dabei gedacht, was sie gefühlt haben. Das Ziel jeder CD-Produktion kann daher nur sein, mit welchen Mitteln auch immer, die Aufmerksamkeit der Hörer für die drei Minuten eines Songs zu fesseln und damit die Nahwelt herzustellen, die eine der zentralen Funktionen der Musik ist. Das geht mit direkter Ansprache durch das vertraute »Du«, aber auch durch die Produktion einer virtuellen körperlichen Intimität: Sex sells. Zeichen der Erotik und der Liebe verhindern, dass das Musikvideo weggezappt, der Song ausgeschaltet wird. Im Alltag eskaliert Kommunikation mit Codes der Erotik. Die Zeichen müssen, damit Kommunikation weiter läuft und Aufmerksamkeit nicht nachlässt, nach und nach eindeutiger, intimer werden. Aus dieser Erfahrung heraus produzieren erotische Zeichen beim Beobachter Spannung und Aufmerksamkeit über eine gewisse zeitliche Distanz hinweg: Hörer und Betrachter erwarten und warten auf mehr.

Die Massenmedien verstärken die Fiktionalität der Mitteilung noch und bewirken durch diesen Schein des Außer-Alltäglichen, dass Peinlichkeit deutlich herab gesetzt wird. Distanz und Anonymität reduzieren Scham (Duerr 1999: 17). Das Gegenüber auf dem Bildschirm erfährt ja nicht, von wem es schamlos angestarrt wird. Entsprechend deutlich muss Erotik in Massenmedien ausfallen, um überhaupt zu provozieren, um einen Unterschied auszumachen zu einem anderen Song anderer Musiker und um Aufmerksamkeit zu erzeugen. Ob die hieraus entstehende Spirale der Entblößung so weit gehen wird, wie es Robbie Williams in seinem Video zum Song »Rock $D J$ « vormacht, der die Häutung des Marsyas bis auf die Knochen weitertreibt, bleibt abzuwarten. Akustisch sind die Möglichkeiten der Darstellung geschlechtlicher Individualität schon längst voll ausgeschöpft. Stöhnsongs sind seit den 1970er Jahren möglich (vgl. den Beitrag von HansJoachim Erwe in diesem Band), aber immer nur in großen zeitlichen Abständen in einzelnen Genres erfolgreich. Eindeutigkeit kann provozieren, wird 
jedoch auch schnell langweilig. Ähnlich bedeutet auch für visuelle Medien die vollständige Entblößung das baldige Ende der Erotik. Pornographie, das Vormachen von Geschlechtsverkehr, vermag keine kommunikative Nahwelt aufzubauen, da der Beobachter nicht mehr direkt angesprochen wird. Zudem taugt sie kaum zur Darstellung von Individualität. Die Mechanik des Geschlechtsverkehrs erlaubt deutlich weniger Varianten als der kommunikative Weg dorthin. Die verbale Beschreibung von Sex schlägt sogar schnell in das Gegenteil von Erotik um. Besonders deutlich wird die Problematik am Beispiel der von der Presse so genannten »Pornorapper « männlichen und weiblichen Geschlechts, in deren Texten es nicht mehr um Erotik geht, sondern vor allem um die Darstellung von Macht mithilfe körperlicher Zeichen.

Für vorgespielte Musik sind Codes der Erotik und der Liebe wichtig. Sie sichern Aufmerksamkeit, indem sie die Nahwelt zwischen Musikern und Hörern weiter verstärken und trotz dieser Gruppenbildung den Prozess der Individuation der Musiker aber auch der einzelnen Hörer verstärken. Andere symbolisch generalisierte Medien, wie z.B. die Macht, haben, wie angedeutet, ähnliche kommunikative Funktionen. Macht allerdings polarisiert als Medium zwischen Herrschendem und Beherrschten und kann in Gesellschaften mit einem egalitären Selbstverständnis noch stärker provozieren als Erotik. Macht hat durch diese Provokation daher eine stärker differenzierende Wirkung. Für Musik des Mainstreams ist das Medium Macht weniger als Liebe und auch weniger als Erotik geeignet. Auch in Zukunft bleiben, wie seit den ältesten mesopotamischen Quellen, Liebe und Erotik das Thema Nr. 1 für eine Musik, die vom »|ch« singt. 


\section{Literatur}

Aristoteles (2005). Politik. Buch VII/VIII. Übersetzt und erläutert v. Eckart Schütrumpf (= Aristoteles. Werke in deutscher Übersetzung 9, IV). Berlin: Akademie.

Aristoteles (2009). Über den Himmel. Übersetzt und erläutert von Alberto Jori (= Aristoteles. Werke in deutscher Übersetzung 12, III). Darmstadt: Wissenschaftliche Buchgesellschaft.

Augustinus (1981). Confessionum Libri XIII. Hg. v. Lucas Verheijen (= Sancti Augustini opera 27). Turnhout: Brepols.

Darwin, Charles (1910). Der Ausdruck der Gemütsbewegung bei dem Menschen und den Tieren. Übers. v. U. Victor Carus. Stuttgart: Nägele \& Dr. Sprosser (6. Aufl.).

Duerr, Hans Peter (1995). Obszönität und Gewalt (= Der Mythos vom Zivilisationsprozeß 3). Frankfurt/M.: Suhrkamp.

Duerr, Hans Peter (1999). Der erotische Leib (= Der Mythos vom Zivilisationsprozeß 4). Frankfurt/M.: Suhrkamp.

Elias, Norbert (1994). Die höfische Gesellschaft. Untersuchungen zur Soziologie des Königtums und der höfischen Aristokratie. Frankfurt/M.: Suhrkamp.

Elias, Norbert (1997). Über den Prozeß der Zivilisation. Soziogenetische und psychogenetische Untersuchungen. 2 Bde. Frankfurt/M.: Suhrkamp.

Feldman, Martha (Hg.) (2006). The Courtesan's Arts: Cross-Cultural Perspectives. New York, Oxford: Oxford University Press.

Frateantonio, Christa (2010). »Bacchanal(ia).«In: Der Neue Pauly. Hg. v. Hubert Cancik und Helmuth Schneider (Antike), Manfred Landfester (Rezeptions- und Wissenschaftsgeschichte). Leiden, Boston: Brill. Online unter: http://www. brillonline.nl/subscriber/entry?entry=dnp_e211000 (Zugriff am 17.6.2010).

Frith, Simon (1998). Performing Rites. Evaluating Popular Music. Oxford, New York: Oxford University Press.

Gembris, Heiner (1995). »Musikalische Interessen und Aktivitäten im Erwachsenenalter. Psychosoziale Funktionen in zwischenmenschlichen Beziehungen.« In: Musikpädagogische Forschungsberichte 1994. Hg. v. Heiner Gembris, RudolfDieter Kraemer und Georg Maas. Augsburg: Wißner, S. 123-133.

Gembris, Heiner (2005). »Vom Nutzen musikalischer Fähigkeiten für die menschliche Entwicklung. «In: Musik: gehört, gesehen und erlebt. Festschrift KlausErnst Behne zum 65. Geburtstag. Hg. v. Claudia Bullerjahn, Heiner Gembris und Andreas C. Lehmann. Hannover: Institut für Musikpädagogische Forschung der Hochschule für Musik und Theater Hannover, S. 235-258.

Guerrieri, Marta Chiara / Giustozzi, Nunzio (2007). Eros [Katalog der Ausstellung Rom, Kolosseum, 3. März-16. September 2007]. Rom: Ministero per i Beni e le Attività Culturali, Soprintendenza Archeologica di Roma.

Haas, Volkert (1999). Babylonischer Liebesgarten. Erotik und Sexualität im Alten Orient. München: Beck.

Hartung, Wolfgang (1982). Die Spielleute. Eine Randgruppe in der Gesellschaft des Mittelalters. Wiesbaden: Franz Steiner.

Helms, Dietrich (2003). »Auf der Suche nach einem neuen Paradigma: Vom System Ton zum System Sound. «In: Pop Sounds. Klangtexturen in der Pop- und Rockmusik. Hg. v. Thomas Phleps und Ralf von Appen (= Texte zur populären Musik 1). Bielefeld: transcript 2003, S. 197-228. 
Helms, Dietrich (2008). »What's The Difference? Populäre Musik im System des Pop. « In: Popmusicology. Perspektiven der Popmusikwissenschaft. Hg. v. Christian Bielefeldt, Udo Damen und Rolf Grossmann. Bielefeld: transcript, S. 75-93.

Helms, Dietrich (2009). „Amor docet musicam. Zu Theorie und Geschichte eines intimen Verhältnisses. «In: Musik - Geschichte(n) - Erzählen. Freundesgabe für Hans-Christian Schmidt-Banse. Hg. v. Hartmuth Kinzler. Osnabrück: Electronic Publishing Osnabrück, S. 79-94.

Johannes von Salisbury (1965). Ioannis Saresberiensis episcopi carnotensis Policratici sive de nvgis cvrialivm et vestigiis philosophorvm libri VIII. Hg. von Clemens C. I. Webb. 2 Bde. Frankfurt: Minerva (Reprint der Ausg. London 1909).

Jourdan-Morhange, Héléne (1945). Ravel et nous: L'homme - l'ami - le musicien. Genf: Éditions du milieu du monde.

Karbusicky, Vladimir (1986). Grundriss der musikalischen Semantik. Darmstadt: Wissenschaftliche Buchgesellschaft.

Kreutz, Gunter (1997). »Musikrezeption zwischen Liebestraum und Loveparade. Sexualität und Sinnlichkeit im Erleben von Musik.« In: Medienpsychologie. Zeitschrift für Individual- und Massenkommunikation 9, H. 4, S. 293-311.

Luhmann, Niklas (1982). Liebe als Passion. Zur Codierung von Intimität. Frankfurt/M.: Suhrkamp.

Luhmann, Niklas (1984). Soziale Systeme. Grundriß einer allgemeinen Theorie. Frankfurt/M.: Suhrkamp.

Luhmann, Niklas (1989). "Wahrnehmung und Kommunikation sexueller Interessen. « In: Sexualitäten in unserer Gesellschaft. Beiträge zur Geschichte, Theorie und Empirie. Hg. v. Rolf Gindorf und Erwin J. Haeberle (= Schriftenreihe Sozialwissenschaftliche Sexualforschung 2). Berlin, New York: de Gruyter.

Mersenne, Marin (1636). Harmonie universelle. [Livre premier. De la voix]. Paris: Sebastien Cramoisy. Faksimile online unter: http://gallica.bnf.fr/ark:/12148/ bpt6k5471093v.r=mersenne.langFR (Zugriff am 20.7.2010).

Mithen, Steven J. (2006). The Singing Neanderthals: The Origins of Music, Language, Mind, and Body. Cambridge, MA: Harvard University Press.

Ortkemper, Hubert (1993). Engel wider Willen. Die Welt der Kastraten. Eine andere Operngeschichte. Berlin: Henschel.

Peirce, Charles S. (2000). »Eine neue Liste der Kategorien.«In: Semiotische Schriften. Hg. von Christian J. Kloesel und Helmut Pape. Darmstadt: Wissenschaftliche Buchgesellschaft, S. 147-159.

Platon (1971). ПOАITEIA. Der Staat. Bearbeitet v. Dietrich Kurz. Griechischer Text von Émile Chambry, dt. Übersetzung von Friedrich Schleiermacher (= Platon. Werke in acht Bänden griechisch und deutsch 4). Darmstadt: Wissenschaftliche Buchgesellschaft.

Platon (1994). Nomoi (Gesetze). Buch I-III. Übersetzung und Kommentar von Klaus Schöpsdau (= Platon. Werke. Übersetzung und Kommentar IX 2). Göttingen: Vandenhoek \& Ruprecht.

Poth, Clodwig (1982). Die Vereinigung von Körper und Geist mit Richards Hilfe. Ein heiterer Liebesroman. Frankfurt/M.: Fischer.

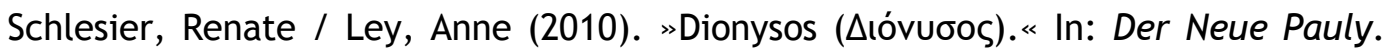
Hg. v. Hubert Cancik und Helmuth Schneider (Antike), Manfred Landfester (Rezeptions- und Wissenschaftsgeschichte). Leiden, Boston: Brill. Online unter: http://www.brillonline.nl/subscriber/entry?entry=dnp_e320270 (Zugriff am 28. 5.2010). 
Schuol, Monika (2004). Hethitische Kultmusik. Eine Untersuchung der Instrumentalund Vokalmusik anhand hethitischer Ritualtexte und von archäologischen Zeugnissen. Rahden: Marie Leidorf.

Wagner, Richard (1992). Tristan und Isolde. Handlung in drei Aufzügen. WWV 90. Teil 2: Zweiter Aufzug. Hg. von Egon Voss und Isolde Vetter (= Wagner. Sämtliche Werke 8, II). Mainz: Schott.

Wallin, Nils L. / Merker, Björn / Brown, Steven (Hg.) (2000). The Origins of Music. Cambridge, MA: MIT.

Zarlino, Gioseffo (1588). Le istituzioni harmoniche. Venedig: Francesco Senese 1562 [1. Aufl. Venedig 1558]. Faksimile online unter: http://imgbase-scd-ulp.ustrasbg.fr/displayimage. $p h p$ ?pid=181593\&fullsize=1 (Zugriff am 20.7.2010).

\title{
Filmographie
}

Williams, Robbie (2000). »Rock DJ« [Musikvideo]. Regie: Vaughan Arnell. London: Emi/Chrysalis.

Edwards, Blake (Regie) (1978). Zehn - Die Traumfrau (Originaltitel: 10). Los Angeles: Orion Pictures.

\begin{abstract}
This paper criticises the biomusicological view that music was introduced as a means of breed selection. Based on Niklas Luhmann's definition of symbolically generalized communication media, this paper defines love, eroticism and music as such media and examines their functions in communication. All three have in common that they produce what Luhmann calls a »Nahwelt « (close-up world) that shuts out the communication of a restricted group of people from their environment; all three media make highly individualized communication probable. The pre-fixed limited time of musical communication gives it a fictional character, a character similar to play. This helps to mark contexts of fictional communication, which allow communication about topics that otherwise are placed under a taboo. Some of these contexts from antiquity to the carnival of modern times are discussed. Music performed before an audience has similar functions in communication as love and eroticism: it makes the display of intellectual and/or bodily individuality possible. The conflict of these two principles - the mind and the body - has influenced the history of musical communication and helped to differentiate the social systems of art and popular music. Five uses of the term »erotic music" are discussed (eroticism symbolized in the composition, eroticism represented through iconic signs [e.g. tone-painting], eroticism in sound, songs with erotic texts and music used to accompany sexual intercourse). Finally reasons are given why popular music is dependent upon erotic communication codes.
\end{abstract}

